\title{
A GMBCG Galaxy Cluster catalog of 55,880 Rich Clusters from SDSS DR7
}

\author{
Jiangang Hao ${ }^{1}$, Timothy A. McKay ${ }^{2,3}$, Benjamin P. Koester ${ }^{4}$, Eli S. Rykoff ${ }^{5}$, Eduardo \\ Rozo $^{6}$, James Annis ${ }^{1}$, Risa H. Wechsler ${ }^{7}$, August Evrard ${ }^{2,4}$, Seth R. Siegel ${ }^{8}$, Matthew \\ Becker $^{9}$, Michael Busha ${ }^{7}$, David Gerdes ${ }^{2}$, David E. Johnston ${ }^{1}$ and Erin Sheldon ${ }^{10}$
}

Received __ ; accepted _

\footnotetext{
${ }^{1}$ Center for Particle Astrophysics, Fermi National Accelerator Laboratory, Batavia, IL 60510

${ }^{2}$ Department of Physics, University of Michigan, Ann Arbor, MI 48109

${ }^{3}$ Department of Astronomy, University of Michigan, Ann Arbor, MI48109

${ }^{4}$ Department of Astronomy and Astrophysics, The University of Chicago, Chicago, IL60637

${ }^{5}$ TABASGO Fellow, Physics Department, University of California at Santa Barbara, 2233B Broida Hall, Santa Barbara, CA 93106

${ }^{6}$ Einstein and KICP Fellow, Kavli Institute for Cosmological Physics, The University of Chicago, Chicago, IL 60637

${ }^{7}$ Kavli Institute for Particle Astrophysics \& Cosmology, Physics Department, and SLAC National Accelerator Laboratory, Stanford University, Stanford, CA 94305

${ }^{8}$ Department of Physics, California Institute of Technology, Pasadena CA 91125

${ }^{9}$ Department of Physics, The University of Chicago, Chicago, IL 60637

${ }^{10}$ Brookhaven National Laboratory, Upton, New York 11973
} 


\begin{abstract}
We present a large catalog of optically selected galaxy clusters from the application of a new Gaussian Mixture Brightest Cluster Galaxy (GMBCG) algorithm to SDSS Data Release 7 data. The algorithm detects clusters by identifying the red sequence plus Brightest Cluster Galaxy (BCG) feature, which is unique for galaxy clusters and does not exist among field galaxies. Red sequence clustering in color space is detected using an Error Corrected Gaussian Mixture Model. We run GMBCG on 8240 square degrees of photometric data from SDSS DR7 to assemble the largest ever optical galaxy cluster catalog, consisting of over 55,000 rich clusters across the redshift range from $0.1<z<0.55$. We present Monte Carlo tests of completeness and purity and perform cross-matching with X-ray clusters and with the maxBCG sample at low redshift. These tests indicate high completeness and purity across the full redshift range for clusters with 15 or more members.
\end{abstract}

Subject headings: Galaxies: clusters, Catalog- Cosmology: observations - Methods: Data analysis, Gaussian Mixture 


\section{Introduction}

One of the most exciting discoveries in physics and astronomy over the past decade is the accelerating expansion of the Universe (Perlmutter et al. 1999; Riess et al. 1998), which has been more recently confirmed by a series of independent experiments (Spergel et al. 2003, 2007; Tegmark et al. 2004; Eisenstein et al. 2005). This cosmic acceleration cannot be explained without exotic physics, for example, modifications to General Relativity (GR), a cosmological constant, or an additional energy component with negative pressure adequate to drive acceleration. Perhaps the simplest possibility, a cosmological constant, is consistent with all available data, although the theoretical challenges with this explanation have not been resolved. If the framework of GR is retained without a cosmological constant, something like dark energy must exist. In an effort to distinguish between these possibilities, studies of expansion history and the growth of structure have become central research topics in physics and astronomy.

One way to test theories of expansion and the growth of structure is to measure the abundance and properties of galaxy clusters. Clusters are the largest peaks in the density field. Their abundance and spatial distribution encode rich information about the Universe (Evrard 1989; Oukbir \& Blanchard 1992), making them sensitive probes for cosmology (Majumdar \& Mohr 2004; Hu 2003; Lima \& Hu 2004, 2005). Cosmological constraints from optically selected galaxy clusters have been carried out recently by Gladders et al. (2007) based on the RCS cluster catalog (Gladders \& Yee 2005a) and Rozo et al. (2007b,a, 2010), based on the maxBCG catalog (Koester et al. 2007a,b).

Galaxy clusters are observationally rich as well. They can be detected and their properties determined using a number of different observables, including X-ray emission from and the Sunyaev-Zeldovich decrement caused by hot intracluster gas, optical and NIR emission from stars in cluster galaxies, and the gravitational lensing distortions imposed 
on background galaxy images by the total cluster gravitational potential. Each probe relies on different aspects of cluster physics and provides different, though often correlated, information about cluster mass and structure. For cluster detection, the different probes have complementary virtues. Cluster X-ray emission and the SZ decrement both require the presence of very hot intracluster gas. This can only be present in very deep potential wells, so these methods only detect the highest mass systems, but are consequently relatively free from projection contamination. Unfortunately, neither very naturally provides information about cluster redshift, so optical follow-up is required. Cluster searches using optical data are more able to identify clusters in three dimensions, obtaining distances as part of cluster detection. Optical selection can identify systems corresponding to much lower mass dark matter halos than methods based on the intracluster gas, but this also results in more serious projection effects. Cluster detection in the optical also benefits from the high signal to noise for individual galaxy detection and large data volumes available in optical surveys.

The existence of a uniformly old stellar population in many cluster galaxies gives them remarkably similar spectral energy distributions which include a strong 4000 angstroms break. As a result, galaxies within clusters are tightly clustered in color as well as space. When the cluster redshift increases, this break shifts across the optical filters, creating a strong correlation between cluster galaxy color and redshift. It has been shown that red-sequence galaxies exist in clusters of varied richness and extend to redshift $z \sim 1.6$ (Bower et al. 1992; Smail et al. 1998; Barrientos 1999; Mullis et al. 2005; Eisenhardt et al. 2005; Papovich et al. 2010). Red sequence galaxies are a very prominent feature of galaxy clusters and thus provide a very powerful means for removing projected field galaxies during cluster detection. As these red sequence galaxies have mostly E and S0 morphologies, dominate the bright end of the cluster luminosity function (Sandage et al. 1985; Barger et al. 1998), and exhibit narrow color scatter ( $\sim 0.05)$, they are also referred to as the E/S0 ridgeline (Visvanathan \& Sandage 1977; Annis et al. 1999). For reviews of 
red sequence galaxies in clusters, refer to Gladders \& Yee (2000), Hao et al. (2009) and references therein.

In this paper, we extend the use of red sequence galaxies and brightest cluster galaxies (BCG) for cluster detection, and develop an efficient cluster finding algorithm which we name the Gaussian Mixture Brightest Cluster Galaxy (GMBCG) method. The algorithm uses the Error Corrected Gaussian Mixture Model (ECGMM) algorithm (Hao et al. 2009) to identify the BCG plus red sequence feature and convolves the identified red sequence galaxies with a spatial smoothing kernel to measure the clustering strength of galaxies around BCGs. We apply this technique to the Data Release 7 of Sloan Digital Sky Survey and assemble a catalog of over 55,000 rich galaxy clusters in a redshift range extending from $0.1<z<0.55$. The catalog is approximately volume limited up to redshift $z \sim 0.4$ and shows high purity and completeness when tested against a mock catalog. The algorithm is very efficient, producing a cluster catalog for the full SDSS DR7 data $\left(\sim 8,000 \mathrm{deg}^{2}\right)$ within 23 hours on a single modern desktop computer.

Cluster finding algorithms are closely related to the properties of the data they are applied to. Therefore, we begin with a general description of the GMBCG algorithm, then add additional features that are particular to its application to the SDSS data. The paper is organized as follows: in $\S 2$, we review de-projection, the major challenge of optical cluster detection, summarizing the de-projection methods used in previous cluster finding algorithms and demonstrating why red sequence color outperforms the others. In $\S 3$, we introduce the major steps of the GMBCG algorithm and compare it with the maxBCG algorithm. In $\S 4$, we introduce the cluster catalog we constructed from the SDSS DR7 using the GMBCG algorithm. In $\S 5$, we evaluate this new DR7 catalog by matching it to catalogs of known X-ray clusters and previously published maxBCG clusters. The completeness and purity of the GMBCG catalog are then also tested against a mock catalog. We conclude 
with a summary of the properties of the GMBCG catalog, along with a discussion of the prospects for using this method on future optical surveys.

By convention, we use a $\Lambda$ CDM cosmology with $h=1, \Omega_{m}=0.3$ and $\Omega_{\Lambda}=0.7$ throughout this paper. Also, we will omit the $h^{-1}$ when describing distances, i.e., we will use Mpc directly instead of $h^{-1} \mathrm{Mpc}$.

\section{Optical Galaxy Cluster Detection and De-projection}

Our goal is to detect galaxies clustered in three spatial dimensions, but we have precise information in only two: RA and DEC. Large uncertainties in galaxy position along the line of sight leads to projections which contaminate richness estimates for all clusters and confuse cluster detection at low richness. Therefore, every optical cluster finding algorithm needs to effectively de-project field galaxies before calculating overdensities in the RA/DEC plane.

The ability to locate the positions of galaxies along the line of sight is limited by the technology available. Over the past 60 years, various algorithms for optical galaxy cluster detection based on photometric data have been employed (Abell 1957; Huchra \& Geller 1982; Davis et al. 1985; Shectman 1985; Efstathiou et al. 1988; Couch et al. 1991; Lidman \& Peterson 1996; Postman et al. 1996; Kepner et al. 1999; Annis et al. 1999; Gladders \& Yee 2000, 2005b; Gal et al. 2000, 2003; Kim et al. 2002; Goto et al. 2002; Ramella et al. 2002; Lopes et al. 2004; Botzler et al. 2004; Koester et al. 2007b; Li \& Yee 2008). ${ }^{1}$ For a recent review of the cluster finding algorithms, see Gal (2006). Though these methods differ

\footnotetext{
${ }^{1}$ When spectroscopic redshifts are available, other algorithms have been developed, for example, Berlind et al. (2006); Yang et al. (2007); Miller et al. (2005). In this paper, we will mainly consider the algorithms based on photometric data.
} 
in many detailed respects, we can roughly classify them according to the de-projection methods they use. In Table. 1, we list the cluster finding algorithms for photometric data of the past two decades and the de-projection methods used.

The de-projection method used by each algorithm is often determined by the properties of the data for which the algorithm was developed. When only single band data were available the major de-projection methods were all magnitude based. However, the broad luminosity function of galaxies makes magnitude a poor indicator of galaxy position along the line of sight. Even so, these methods are quite effective for detecting massive clusters. Unfortunately, they cannot maintain good purity and completeness for clusters with low or intermediate richness. Moreover, the contamination of cluster richness induced by projection also creates large scatter in the richness-mass relations derived from these methods.

Multi-band digital imaging technology greatly alleviates the projection effects that plagued optical galaxy cluster detection for decades. In a precise multi-band sky survey, we have magnitude information from more than one band, allowing better reconstruction of the galaxy spectra. Even the crude Spectral Energy Distribution (SED) information provided by colors provides very effective information for locating galaxies along the line of sight.

The red sequence, or E/S0 ridgeline, which defines cluster galaxies, has a very narrow color scatter $(\sim 0.05)$ and a slightly tilted color magnitude relation, the study of which has a long history, e.g. (Visvanathan \& Sandage 1977; Bower et al. 1992; Gladders et al. 1998; López-Cruz et al. 2004; Blakeslee et al. 2003, 2006; De Lucia et al. 2007; Stott et al. 2009; Mei et al. 2009; Hao et al. 2009). This color information is the primary tool to determine the position of galaxies along the line of sight.

There are basically two ways to de-project galaxies using multi-color data: use the colors to obtain photometric redshifts and then de-project using these redshifts, or 
Table 1: Summary of optical cluster finding algorithms for photometric data

\begin{tabular}{|c|c|c|}
\hline Algorithm & Type of data applied & De-projection method \\
\hline Percolation $^{a}$ & Single band/Simulation & Magnitude/photo- $z$ \\
\hline Smoothing Kernels ${ }^{b}$ & Single band & Magnitude \\
\hline Adaptive Kernel $^{c}$ & Single band & Magnitude \\
\hline Matched Filter ${ }^{d}$ & Single band & Magnitude \\
\hline Hybrid and Adaptive Matched Filter ${ }^{e}$ & Single band & Magnitude/photo- $z$ \\
\hline Voronoi Tessellation ${ }^{f}$ & Single band & Magnitude \\
\hline Cut-and-Enhance ${ }^{g}$ & Single band & Magnitude \\
\hline Modified Friends of Friends ${ }^{h}$ & Multi-band & Photo- $z$ \\
\hline $\mathrm{C} 4^{i}$ & Multi-band & All Colors \\
\hline Percolation with Spectroscopic redshift ${ }^{j}$ & Multi-Band & Spectroscopic Redshift \\
\hline Cluster Red Sequence ${ }^{k}$ & Multi-band & Red sequence \\
\hline $\mathrm{MaxBCG}^{l}$ & Multi-band & Red sequence \\
\hline GMBCG & Multi-band & Red sequence \\
\hline
\end{tabular}

${ }^{a}$ Huchra \& Geller (1982); Davis et al. (1985); Efstathiou et al. (1988); Ramella et al. (2002)

${ }^{b}$ Shectman (1985)

${ }^{c}$ Gal et al. $(2000,2003)$

${ }^{d}$ Postman et al. (1996)

${ }^{e}$ Kepner et al. (1999); Kim et al. (2002); Dong et al. (2008)

${ }^{f}$ Kim et al. (2002); Lopes et al. (2004)

${ }^{g}$ Goto et al. (2002)

${ }^{h}$ Li \& Yee (2008)

${ }^{i}$ Miller et al. (2005)

${ }^{j}$ Berlind et al. (2006)

${ }^{k}$ Gladders \& Yee $(2000,2005 b)$

${ }^{l}$ Annis et al. (1999); Koester et al. (2007a,b) 
use the red sequence to detect clustering directly in color space. The first approach is straightforward in principle, but more complex in practice. There are many machine learning algorithms (Oyaizu et al. 2007; Gerdes et al. 2009) that can be used to assign photo- $z \mathrm{~s}$ based on the multi-band colors/magnitudes. However, these methods are limited by the available training set of spectroscopic redshifts. For galaxies that are similar to the training set, reconstructed photo- $z$ s can reach a precision of $\sim 0.03$ (Oyaizu et al. 2007). However, for galaxies that are not represented in the training set, photo-zs can be very imprecise and biased.

To get a sense of how photo- $z$ s perform for all galaxies, we can simply compare the results of two different estimators. Take the neural network photo- zs for SDSS data (Oyaizu et al. 2007) as an example. There are two well-tested estimators provided in the SDSS catalogs, labeled photozd1 and photozcc2. The photozd1 is obtained by training only on magnitudes, while photozcc2 is obtained by training only on colors. In Figure 1, we compare photo- $z$ s based on these two estimators. The difference of the two photo-zs has a standard deviation of $\sim 0.1$. For a typical cluster, with a velocity dispersion of $900 \mathrm{~km}$ $s^{-1}$, the dispersion between galaxy redshifts is \pm 0.003 , much smaller that the precision possible from photo- zs alone. Therefore, though it is a lot better than the magnitude based de-projection, photo- $z$ de-projection will still be insufficient to remove projection effects entirely, especially when training sets remain limited.

As an alternative, we may stay closer to the data and look for clustering directly in color space. Red sequence galaxies in low redshift clusters display a scatter in $g-r$ color of $\sim 0.05$. Most importantly, a tight cluster red sequence accompanied by a BCG presents a pattern exhibited only by clusters and not found in field galaxies. Therefore, directly looking for the red sequence plus BCG feature provides a powerful way to improve cluster detections. It is this approach which we follow in the GMBCG method. 

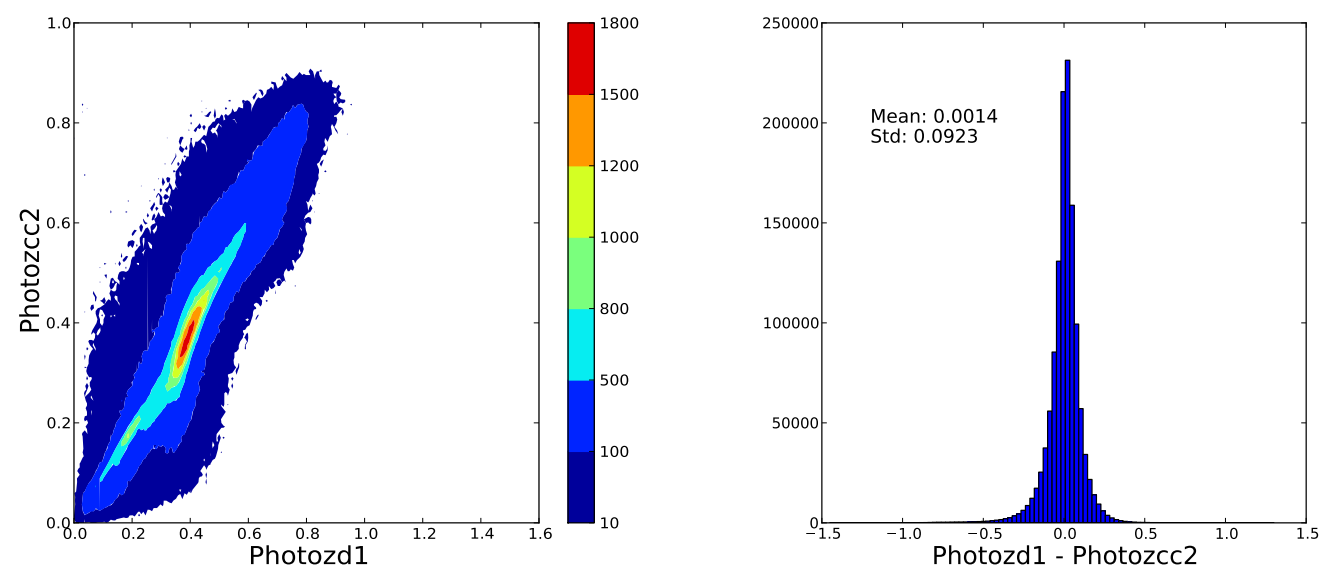

Fig. 1. - Scatter between two well-trained photo- $z$ estimators. Two neural network algorithms from Oyaizu et al. (2007) (photozd1, which uses magnitudes only, and photozcc2, which uses galaxy colors) applied to SDSS DR6 data are compared. Left panel plots the two estimators against each other for the full sample, right panel shows the scatter between the two estimators. Although the algorithms are well tuned using existing spectroscopic data, the two photo- $z$ s have an rms difference of $\sim 0.1$. 


\section{Details of the GMBCG Algorithm for Optical Cluster Detection}

\subsection{Overview}

As pointed out in the previous section, the BCG plus red sequence pattern is a unique feature of galaxy clusters. We therefore make identifying this feature a key step in our cluster finding algorithm. The distribution of galaxy colors in a cluster can be well

approximated by a mixture of two Gaussian distributions(Hao et al. 2009). The redder and narrower Gaussian distribution corresponds to the cluster's red sequence, while the bluer and wider one includes both foreground and background galaxies along with the "blue cloud" cluster members. In Figure 2, we show the galaxy color distribution around two real clusters and the corresponding color magnitude relation. If there is no cluster, then the color distribution in a given patch of sky will be well represented by a single Gaussian with a wide width. Fitting the color distribution with mixture of Gaussian distributions is well suited for our purpose. A complication in our case is that the measurement errors of the colors are not negligible and proper modelling of them is essential for the detection of red sequence. The traditional Gaussian Mixture Model (GMM) does not consider the measurement errors and we therefore use an error corrected GMM to developed in our earlier work (Hao et al. 2009).

As long as we effectively isolate red sequence galaxies, we reduce the problem of cluster finding to a clustering analysis on the ra/dec plane. One can then use either parametric (such as convolving with a model kernel) or non-parametric (such as Voronoi Tessellation) methods to analyze the strength of the clustering signal. When we apply such a scheme to data spanning a wide redshift range there are three other complications to consider.

First, as redshift increases the red sequence shows up in different colors. This is mainly 

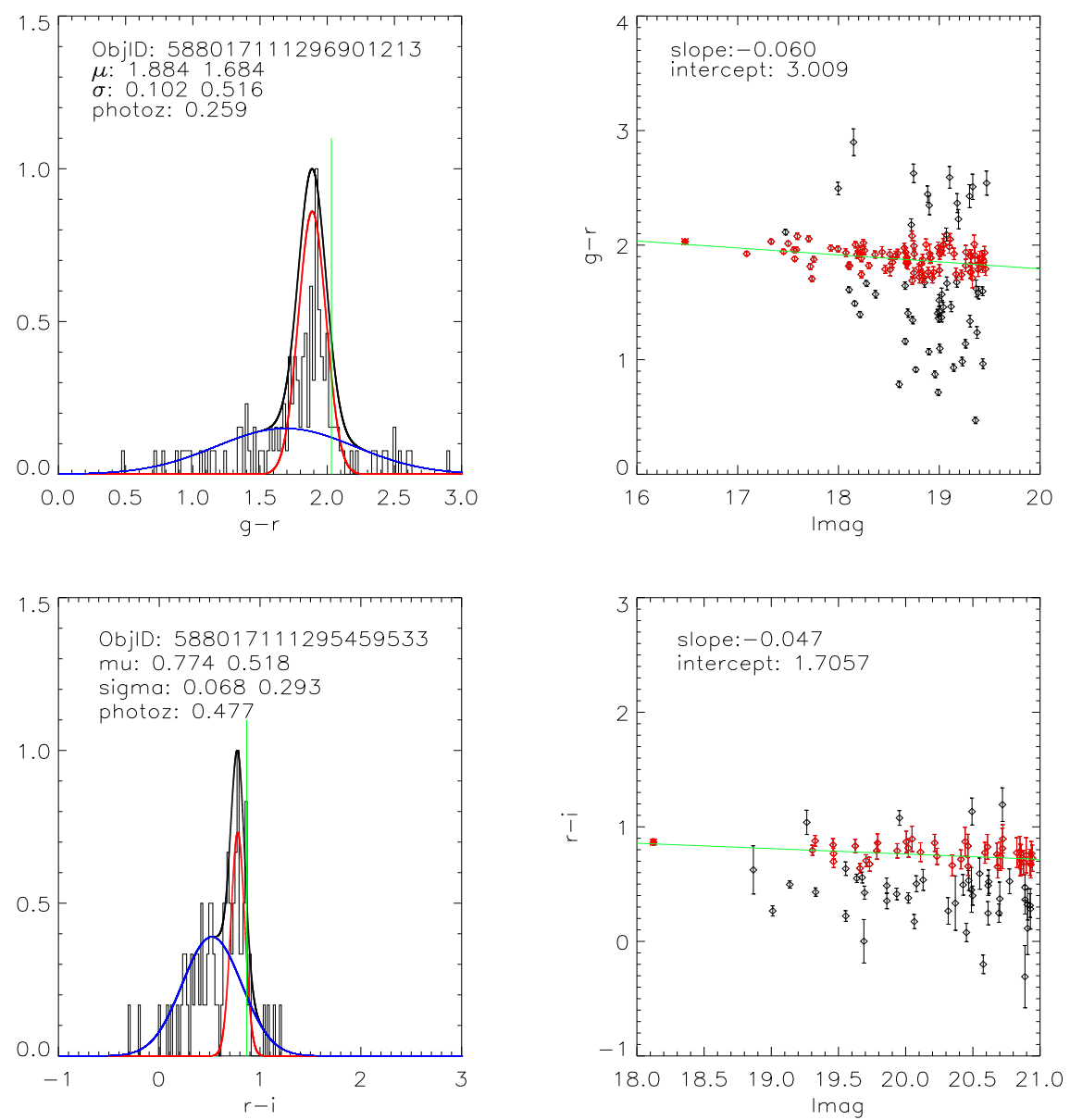

Fig. 2.- Color distributions and color-magnitude relations around two representative clusters. Top Left Galaxy $g-r$ color distribution around a cluster overlaid with a model constructed of a mixture of two Gaussian distributions. The red curve corresponds to the red sequence component while the blue one corresponds to the sum of background galaxies and blue cluster members. The green vertical line indicates the color of the BCG. $\mu$ and $\sigma$ are the means and standard deviations of the two Gaussian components. Top right Color-magnitude relation for the same galaxies. Galaxies within the $2 \sigma$ clip of the red sequence component are shown with red points; the green line indicates the best fit slope and intercept of this red sequence. The left most red point is the BCG. The bottom two panels shown the same plots for a second, higher redshift cluster, where the color used is $r-i$ instead of $g-r$. 
a result of the $4000 \AA$ break shifting across the filters. Because of this effect, the most informative color will vary as redshift increases. For the set of SDSS filters, the relation between red sequence color and redshift is given in Table 2 .

Beyond $z \sim 1.0$, one needs near infra-red color information, from bands like $\mathrm{Y}, \mathrm{J}$, $\mathrm{H}$, or K. Therefore, when detecting clusters in data spanning a wide redshift range, it is necessary to determine which ridgeline color we should examine. Since we will be searching for the red sequence around candidate BCGs, we adopt the BCG's photo- $z$ as a good estimator of cluster's redshift. BCGs are bright, making their photo- $z$ s generally much better determined than more typical galaxies. As we discuss in $\S 3.3$, the precision of BCG photo- zs is sufficient to determine which red sequence color should be examined around a given BCG. This does require a determination of the photo- $z$ for every candidate BCG before proceeding.

A second complication for cluster finding across a broad redshift range is the increased chance of overlapping clusters, one at low redshift and another at relatively high redshift. Such an overlap will complicate the distribution in color space, turning it from bimodal to tri-modal or even more. To reduce the possibility of this occurring, we apply a broad photo- $z$ window (such as \pm 0.25 in photo- $z$ ) to select potential member galaxies before searching the color distribution. The available photo- $z$ precision is adequate for this

Table 2: Red sequence color in different redshift ranges for SDSS filters

\begin{tabular}{cc}
\hline \hline Ridgeline color & Redshift range \\
\hline$g-r$ & $0.0 \sim 0.43^{a}$ \\
$r-i$ & $0.43 \sim 0.70$ \\
$i-z$ & $0.70 \sim 1.0$ \\
\hline
\end{tabular}

${ }^{a}$ Although the 4000 Ảbreak starts shifting into SDSS $\mathrm{r}$ band at $z \sim 0.36$, we observed that $g-r$ color is still better than $r-i$ color for detecting red sequence up to redshift 0.43 . 
purpose. In addition to photo- $z$ clips, we also apply luminosity cuts and require the potential member galaxies to be brighter than $0.4 L^{*}$, where $L^{*}$ is the characteristic luminosity in the Schechter luminosity function. For our application, the $i$-band apparent magnitude corresponding to $0.4 L^{*}$ as a function of redshift is shown in the lower right panel of Figure 3. We adopted this from Annis et al. (1999) and Koester et al. (2007a). Selecting potential member galaxies by cutting on photo- $z$ and luminosity is very effective at simplifying the color space structure around the target galaxies. In addition to this, the $0.4 L^{*}$ cut allows us to measure a consistent richness at different redshift ${ }^{2}$.

The third complication concerns defining a consistent measurement of richness across more than one color. Red sequence galaxies selected from different color bands have different degrees of contamination from the background. This is a fundamental limit of all color-based red sequence selection methods, though it has a relatively minor effect on our cluster detection. Once a cluster catalog is produced, we will need to further calibrate the richness measured from different color bands using other means, such as gravitational lensing analysis (Sheldon et al. 2007; Johnston et al. 2007). In the present work, we just adjust the richness definitions to result in a smooth transition between filters.

\subsection{Brightest Cluster Galaxies as Cluster centers}

Brightest cluster galaxies (BCGs) reside near the cluster center of mass, and provide important clues to other observational features of clusters. Choosing the BCG as the center in a cluster finding algorithm has good physical, algorithmic, and computational motivations. The major physical motivation for focusing on the BCG is that the central galaxy in a cluster (the one which resides near the bottom of the cluster potential well) is

\footnotetext{
${ }^{2}$ For the SDSS DR7 data, the $0.4 L^{*}$ can keep a consistent richness up to redshift 0.4 .
} 

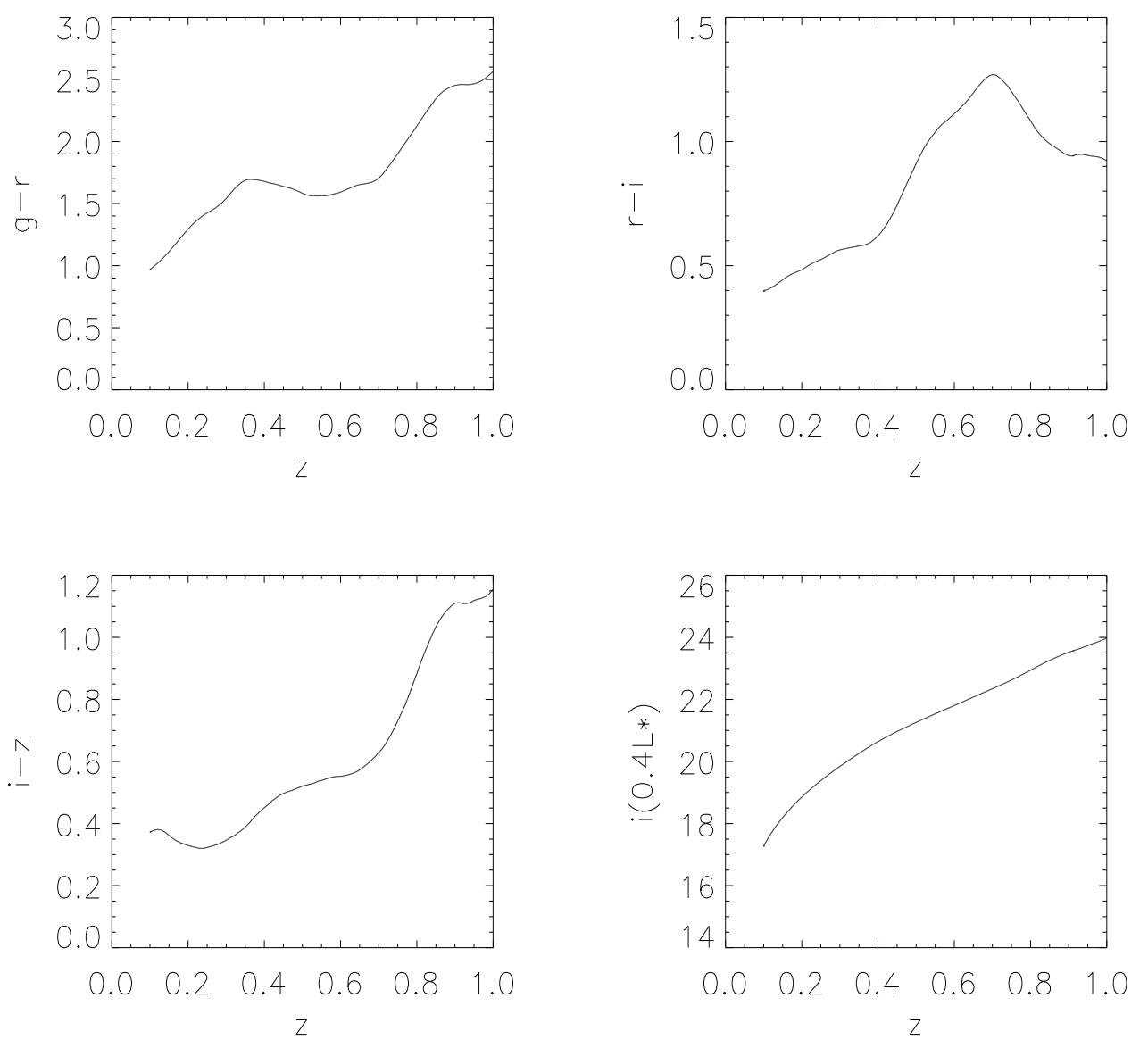

Fig. 3.- The top two and bottom left panels are the color evolution based on a color model of the red sequence galaxies (Koester et al. 2007a). The bottom right panel is the I band apparent magnitude corresponding to $0.4 L^{*}$ at different redshifts. 
very often the brightest galaxy in the cluster. This BCG is then coincident with the region with the deepest potential traditionally identified in theory as the center of a cluster. To the extent that this is true, using the BCG as the cluster center simplifies precise comparisons between observations and theory, although the extent to which the brightest galaxy is always at the center, and the extent to which the most central galaxy is at the center of the dark matter potential well, are still areas of investigation.

In an algorithmic sense, the BCG helps to distinguish among the bright galaxies typically found near the cluster center. Such galaxies are all similarly clustered, and the choice of a cluster center is thus somewhat dominated by noise. The uniqueness of BCGs, including their often cD-like morphologies, acts as a "noise damper" for positioning cluster center. Computationally, BCGs are bright and have well-determined photo-zs, and the combination of these phenomena boosts the efficiency of cluster detection by omitting searches around intrinsically faint galaxies that dominate the luminosity function. These motivating factors underscore the fact that while BCGs do not drive the identification of clusters in the current algorithm, they play an important fine-tuning role that minimizes the need for downstream modelling in cosmological analyses.

\subsection{Red Sequence Color Selection}

A filter combination tuned to the selection of red-sequence galaxies at a given redshift is of utmost importance. In our algorithm, we use the photo- $z$ of the BCG to determine which color to choose. For SDSS filters, we list the corresponding red shift ranges for different colors in Table 2. In principle, the wrong color can be chosen for a cluster due to an inaccurate BCG photo- $z$. In practice this is not a serious problem; the photo- $z \mathrm{~s}$ for BCGs are usually well determined ( $\sim 0.015$ for SDSS DR7, see $\S 4.2 .2$ ). Redshifts that place the $4000 \AA$ break near the border of the filters are also a cause for concern, as 
they can confuse the filter choice. However, near the filter transitions, the BCG plus red sequence pattern is apparent in both adjacent colors. For example, for a rich cluster located at $z=0.42$, which falls in the transition region from the SDSS $g$ band to the $r$ band, the

combined red sequence and BCG features can be still be easily captured in either the $g-r$ or $r-i$. This ambiguity can impact the richness estimates for clusters near the transition between filters (see $\S 4.2$ ), but does not result in issues for cluster detection for the richness range considered in the current work.

\subsection{Red Sequence Detection}

\subsubsection{Cluster Member Galaxy Selection}

The sizes of clusters are varied, increasing substantially with mass. Therefore, using a scaled aperture is preferred for keeping a consistent richness estimation. Ideally for a candidate cluster, a series of different aperture radii should be examined and chosen by maximizing S/N. However, this can be computationally expensive. As a substitute, we take a two-step approach similar to Koester et al. (2007b), which attempts to deal with this fact: first, we measure the richness of the cluster using a fixed metric aperture; then we scale the radius based on our measured fixed aperture richness and remeasure everything using the scaled aperture size. The following describes the exact implementation for the current work. 


\subsubsection{Fixed Aperture Membership and Richness}

For a candidate BCG, we identify cluster members using a multi-step process. We draw a $0.5 \mathrm{Mpc}$ circle around the candidate BCG at its photo- $z^{3}$ and select all galaxies fainter than the candidate $\mathrm{BCG}$, but brighter than the $0.4 L^{*}$ cut at the relevant photo- $z$. Using the filter combination relevant for the BCG, we use the Gaussian Mixture Model to fit the distribution of the colors of all the galaxies selected above. To remove possible overlap of two or more clusters along the line of sight, we consider only galaxies within a photo- $z$ window of \pm 0.25 around the BCG. To determine the appropriate number of Gaussian components for the fitting the color distribution, we calculate the Akaike Information Criterion (AIC Akaike 1974). Around a cluster, AIC normally chooses two Gaussian as best fit, one narrow and one broad, and the former is chosen as the red sequence as it sits red-ward. Using the fixed $0.5 \mathrm{Mpc}$ aperture it is, however, possible that the field of view is dominated by a large cluster and therefore the best fit to the color distribution is a single Gaussian representing the red-sequence. This highlights the need for a scaled aperture (in this case, enlarged) which would include more background galaxies and push the fitting towards two color components.

Next, for the two mixture case, we need to determine to which Gaussian component the candidate BCG belongs. We compare its corresponding likelihoods of the candidate BCG's color belonging to each of the two Gaussian components and assign the most likely Gaussian

\footnotetext{
${ }^{3}$ The BCG's photo-z is a good estimator of the cluster redshift (see Figure 11). One might use the weighted average of the member galaxy photo-zs in the expectation that the $\sqrt{(N)}$ averaging would provide a more accurate estimated redshift. This is true, however, only when there is no systematic bias in the member photo-zs. In current practice there are often systematic errors in these members related to their being fainter and yet just as red as the BCG. There will always be the issue that they have lower signal/noise than the BCG.
} 
component to the candidate BCG. If this Gaussian component is wider than than the other Gaussian component, we flag this candidate BCG as a field galaxy and remove it from the searching list for next steps. For the case where there is only one Gaussian component, we impose a threshold on its width, beyond which we do not deem it suggestive of a red sequence and remove the corresponding candidate BCG from consideration. Extensive testing on rich clusters in the SDSS sets a color width of 0.16 (about twice the intrinsic width) as an appropriate threshold in both the $g-r$ and $r-i$ colors.

Following this process, we consider only the candidate BCGs with an appropriate red sequence measured. All the galaxies whose colors are within \pm 2 standard deviations of the mean of the corresponding Gaussian component are flagged as members. The number of

member galaxies selected this way is denoted as $N_{\text {gals }}^{0.5 M p c}$. The $\pm 2 \sigma$ cut corresponds roughly the level where the background likelihood dominates over cluster likelihood. It is shown elsewhere that indeed the two component Gaussian Mixture Model can reliably pick up the correct peak in color space as verified by simulations (Hao et al. 2009).

\subsubsection{Scaled Aperture Size and Richness}

Scaled apertures are required to measure clusters of different sizes. To select the appropriate aperture, we assume there is a scaling relation between the aperture and the richness we measured with $0.5 \mathrm{Mpc}$ aperture, as motivated by Hansen et al. (2007).

$$
R_{\text {scale }}=N\left(N_{\text {gals }}^{0.5 M p c}\right)^{P}
$$

where $N$ and $P$ are the normalization and power respectively, which need to be set so that the resulting $R_{\text {scale }}$ corresponds roughly to the relevant value of $R_{200}$. To determine the scaling relation, we measure the $N_{\text {gals }}^{0.5 M p c}$ for maxBCG clusters (Koester et al. 2007a). For every maxBCG cluster, there is a $R_{200}^{\text {lens }}$ measured, interior to which the mean mass density 
of the cluster is 200 times of the critical energy density. This $R_{200}^{\text {lens }}$ is measured based on an exhaustive weak lensing analysis (Johnston et al. 2007; Hansen et al. 2007). We find that $N_{\text {gals }}^{0.5 M p c}$ and the corresponding $R_{200}^{\text {lens }}$ follow a simple relation,

$$
R_{\text {scale }}=0.133 \times\left(N_{\text {gals }}^{0.5 M p c}\right)^{0.539}
$$

where $R_{\text {scale }}$, measured in Mpc, plays the role of the $R_{200}^{\text {lens }}$ in Johnston et al. (2007) and Hansen et al. (2007). Once we have the scaled aperture, we repeat the procedure for the fixed aperture richness measurement, substituting the corresponding scaled aperture for 0.5 Mpc. The corresponding richness is denoted as $N_{\text {gal }}^{\text {scaled }}$, and is used as the primary estimate of richness for the cluster catalog.

\subsubsection{GMM vs ECGMM and Weighted Richness}

In this prescription for cluster member selection, we rely on the detection of the red sequence as well as the measurement of its width. The Gaussian Mixture Model (GMM) and its generalization with error correction (ECGMM) are well-suited to detecting the red sequence in a cluster. An unbiased measurement of the evolution of the red sequence and its width requires the ECGMM (Hao et al. 2009). However, as the measurement errors increase, we cannot simply select member galaxies using ECGMM with a $2 \sigma$ ( $\sigma$ is the standard deviation of the Gaussian component corresponding to the red sequence) cut in a consistent way. GMM does give consistent membership selection. This is mainly due to the fact that the ECGMM measures "true" ridgeline width while our cuts are made in terms of the observed colors. However, as the measurement error increases (e.g. at higher redshift), GMM struggles to discern the correct number of Gaussian components, as the measurement errors "blur" the color distribution. In this case, GMM will more likely favor a single Gaussian component over two based on AIC, but ECGMM more accurately recovers the correct number of mixtures because it properly models the measurement errors. 
On the other hand, we can also measure a weighted richness. When we apply GMM (ECGMM) to fit the color distribution, each Gaussian component has a weight from the fitting. This weight quantifies how much of the total population is from the corresponding Gaussian component. By multiplying the relative weight of the cluster component to the total number of galaxies in the field, we measure the weighted richness. It turns out that this weighted richness correlates better with the true richness of the cluster when they are well measured ${ }^{4}$. To demonstrate this, we performed some Monte Carlo tests. First, we generate the mock colors from two Gaussian distribution, one corresponds to the background and another corresponds to the cluster. We fix the number of galaxies in the background component as 40 while vary the number of galaxies in cluster component from 10 to 70 with increment of 5 . Then, we generate the measurement errors from a uniform distribution scaled by a noise level ( 0.1 and 0.2 respectively in our case). The mock color will be updated by adding realizations from a Gaussian distribution with the width specified by the measurement errors.

For each given mock cluster richness, we repeat the above procedure 100 times and obtain a richness and weighted richness measurements using GMM/ECGMM each time. In Figure 4, we plot our measured mean richness (NFound) and mean weighted richness (Weighted NFound) vs the true richness (NTrue) at different measurement noise level.

Based on these analyses, we conclude that GMM can give better richness counts while ECGMM can give better weighted richness. Therefore, in practice, we will use a hybrid of both GMM and ECGMM. We firstly detect the red sequence using ECGMM and measure the weighted richness, and then we use GMM with fixed number of mixtures (according to the results of ECGMM) to do a follow-up measurement and select the red sequence

\footnotetext{
${ }^{4}$ Note if there is only one Gaussian component, this weighted richness does not make sense.
} 

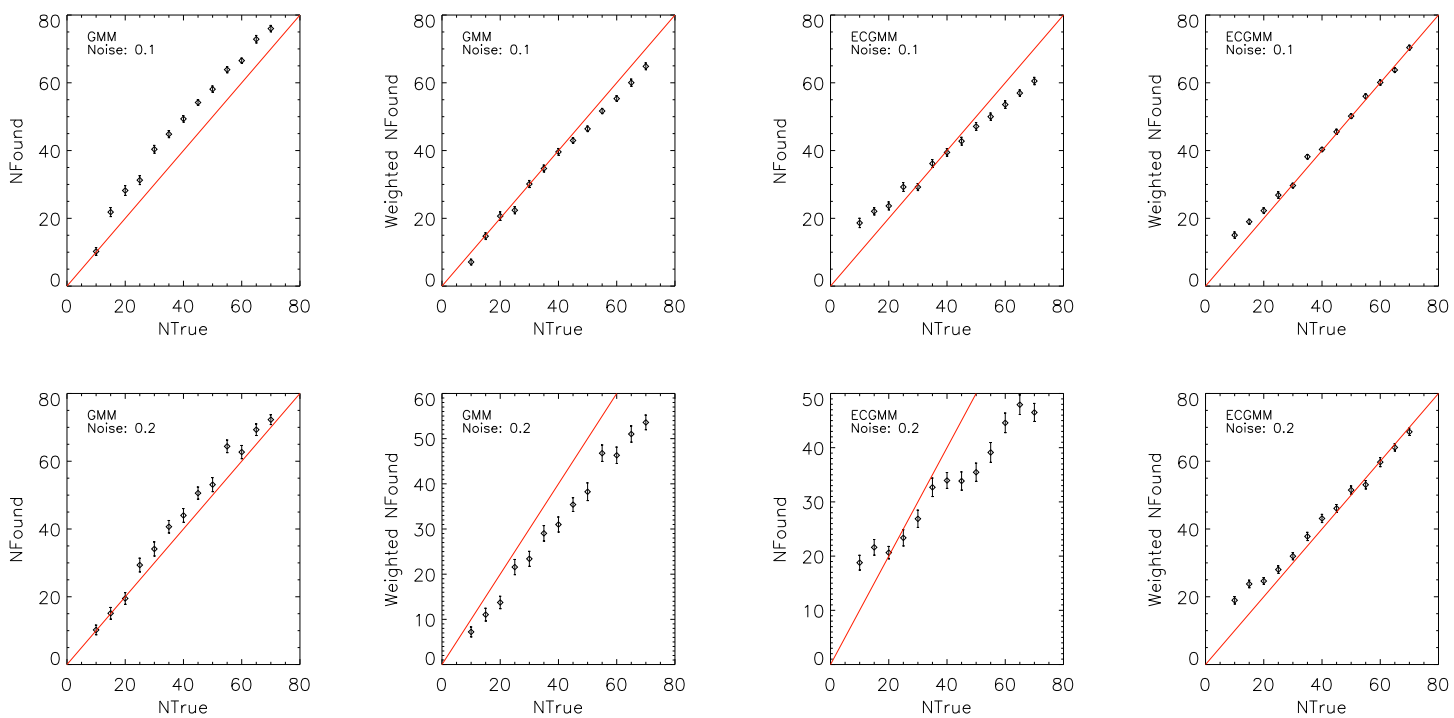

Fig. 4. - Reconstruction of richness using GMM and ECGMM at noise level 0.1 and 0.2. The noise on the plot indicate the scale we used to generate the mock measurement errors. GMM results in better number counts reconstruction, while ECGMM gives better weighted richness as measurement noise varies. 
members.

\subsection{Clustering Strength}

We now have sufficient machinery to detect red sequence around a given BCG candidate. If there is red sequence detected, it is still possible that the candidate BCG is, e.g., a bright foreground galaxy, and does not belong to the red sequence. Criteria must be chosen to determine the association of a candidate BCG with the identified red sequence. We thus consider it to be "associated" with the red sequence if its color lies within 3 standard deviations of the peak of the identified red sequence Gaussian.

Next, we quantify the strength of spatial clustering in the ra/dec plane by convolving the selected members with a projected NFW (Bartelmann 1996; Navarro et al. 1997; Koester et al. 2007b) radial kernel. It is worth noting that the type of kernel used is not as important as its scale, which has been revealed by statistical kernel density analyses (Silverman 1986; Scott 1992). Therefore, the specific kernel does not significantly bias the detection of clusters that deviate from the kernel shape. We introduce the clustering strength as

$$
S_{\text {cluster }}=\sum_{k=1}^{N_{g}} \Sigma\left(x_{k}\right)
$$

where $N_{g}$ is the total number of member galaxies and

$$
\Sigma(x)=\frac{2 \rho_{s} r_{s}}{x^{2}-1} f(x),
$$

$r_{s}=r_{200} / c$ is the the scale radius, $\rho_{s}$ is the projected critical density, $x=r / r_{s}$ and

$$
f(x)= \begin{cases}1-\frac{2}{\sqrt{x^{2}-1}} \tan ^{-1} \sqrt{\frac{x-1}{x+1}} & x>1 \\ 1-\frac{2}{\sqrt{1-x^{2}}} \tanh ^{-1} \sqrt{\frac{1-x}{x+1}} & x<1 \\ 0 & x=1 \\ 0 & x>20 .\end{cases}
$$


Similar to Koester et al. (2007b), we choose $r_{s}=150 \mathrm{kpc}$, regardless of richness. The clustering strength parameter $S_{\text {cluster }}$ is essentially the height of the peak of the smoothed red sequence density field at the position of the BCG.

\subsection{Luminosity Weighted Clustering Strength}

In addition to the clustering strength parameter introduced in the preceding section, we also measure another luminosity weighted clustering strength $S_{\text {cluster }}^{\text {lum }}$. The measurement is similar to $S_{\text {cluster }}^{\text {strength }}$ except that a luminosity weight $\left(W_{\text {lum }}\right)$ is attached to each galaxy. The luminosity weight is simply defined as the ratio of each galaxy's $i$-band magnitude to the $i$-band magnitude corresponding to $0.4 L^{*}$ at the candidate cluster BCG's redshift.

$$
S_{\text {cluster }}^{\text {lum }}=\sum_{k=1}^{N_{g}} \Sigma\left(x_{k}\right) \times W_{\text {lum }}(k)
$$

The advantage of introducing such a measure is that its ratio to the non-luminosity weighted $S_{\text {cluster }}$ is a good indicator of whether the candidate BCG is a contaminating bright star. This forms an important double check of the star/galaxy separation of the input catalog, which is a minor, but non-negligible source of contamination.

\subsection{Implementation of the Algorithm}

With all the quantities calculated from the above definitions, the implementation of the cluster selection is straightforward. There are basically three steps:

1. For every galaxy in the catalog, evaluate the clustering strength $S_{\text {cluster }}$ inside a 0.5 Mpc searching aperture. This $S_{\text {cluster }}$ is calculated using galaxies fainter than the candidate BCG and belonging to the identified red sequence. 
2. Percolation procedure: rank the candidate BCGs by their clustering strength and remove candidates from the BCG list if they are identified as "members" of another candidate BCG with higher clustering strength. Figure 6 illustrates the distribution of clustering strength around a candidate BCG.

3. Repeat the above process and finally obtain a list of BCGs and their cluster members. Based on the richness measured in $0.5 \mathrm{Mpc}$, one calculates a scaling $R_{\text {scaled }}$ for every BCG. Then processes 1) - 2) are repeated by changing the searching aperture to $R_{\text {scaled }}$ from $0.5 \mathrm{Mpc}$. This concludes the search and completes the final list of BCG members and BCGs with scaled richness $N_{\text {gals }}^{\text {scale }}$.

The procedures are summarized as a flowchart in Figure 5.

\subsection{Post Percolation Procedure}

The above process is essentially a process of detecting the peaks of the smoothed density field, where the height of the peaks is measured by $S_{\text {cluster }}$. In Figure 6, we show the $S_{\text {cluster }}$ measured around Abell 1689.

In this cluster finding process, the center of the cluster is assumed to be the brightest cluster galaxy. Therefore, it is possible that several higher peaks (quantified by $S_{\text {cluster }}$ ) are identified in the field of a brightest cluster galaxy and survive the previous percolation procedure. Multiple peaks must be identified and merged into one cluster using some criteria. This process is deemed "post percolation", in contrast to the previous percolation procedure. The major motivation for not directly blending the peaks during the cluster finding process is the need for additional flexibility in both merging the peaks and avoiding "over-percolation" the true BCGs by some bright stars. Perhaps most importantly, the sub-peaks are indicators of potential cluster sub-structure, and probe the internal structures 


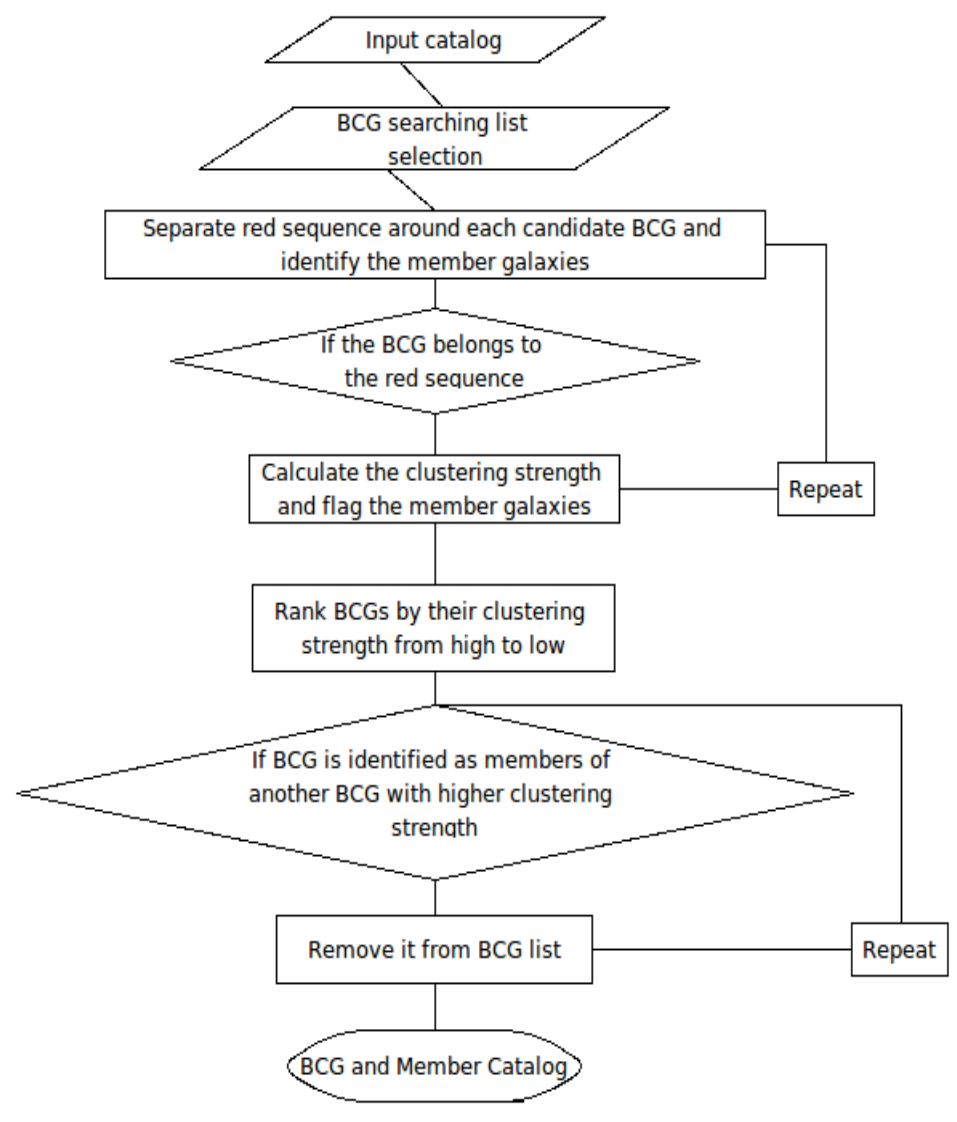

Fig. 5. - Flowchart for the implementation of the GMBCG algorithm 
of clusters.

We settle on the following post-percolation prescription: for a given candidate BCG (denoted as A), we identify a cylindrical region in the ra/dec plane and redshift space around the BCG (A). The radius of the cylinder is $R_{\text {scale }}$ of $\mathrm{BCG}(\mathrm{A})$, and the height is specified by the BCG (A)'s photo- $z \pm 0.05$. Then, if another candidate BCG (denoted as B) falls inside this cylinder and BCG (B) is fainter than BCG (A) but BCG (B)'s clustering strength is not more than 4 times of that of BCG (A), we will merge BCG (B) into BCG (A). Setting the clustering strength threshold of BCG (B) at a level of 4 times more than that of BCG (A) avoids merging a true BCG into a very bright galaxy. The value 4 is obtained explicitly by testing in known situations in the SDSS, where bright foreground objects (e.g. stars) confuse identification.

\subsection{Comparison with MaxBCG Algorithm}

It is interesting to explore the major differences between the GMBCG and maxBCG algorithms (Koester et al. 2007b). maxBCG is a matched filter based algorithm with an additional filter from the red sequence colors. Using this algorithm, a large optical cluster catalog has been created (Koester et al. 2007a), which has high purity and completeness based on tests on both a Monte Carlo catalog and a N-body mock catalog.

The difference between GMBCG and maxBCG can be summarized in three major respects:

1. maxBCG is a generalized matched filter algorithm with the inclusion of a color filter in addition to radial and luminosity filters. It varies the filter at a grid of testing redshifts to maximize the match to a model filter. The redshift at which the model

filter maximizes the match with data is selected as the redshift of the cluster. GMBCG 
does not maximize the match for a redshift dependent filter. It uses a statistically well-motivated mixture model to identify the red sequence plus BCG feature. The radial NFW kernel serves as a smoothing kernel rather than a model filter. Therefore, GMBCG will be less biased against clusters that do not follow the assumed model filter in maxBCG.

2. maxBCG assumes an average ridgeline redshift model for all clusters while GMBCG does not assume any model as a priori. It uses the Gaussian Mixture Model to detect the red sequence and background in a cluster by cluster way. The advantage is that it automatically adjusts the cluster and background parameters across a wide redshift range.

3. In the maxBCG algorithm, the photo- zs of the clusters are estimated as a part of the execution of the algorithm. In GMBCG, photo-zs are obtained from other methods such as neural networks, nearest neighbour polynomial, etc. A photo- $z$ can also be estimated based on the measured red sequence colors as a by product.

For these reasons, GMBCG is more easily extendible to a wide redshift range and less biased against atypical clusters.

\section{GMBCG catalog For SDSS DR7}

In this section, we apply the GMBCG algorithm to the Data Release 7 of the Sloan Digital Sky Survey (SDSS DR7), and construct an optical cluster catalog of more than 55,000 rich clusters across $0.1<z<0.55$. To check the quality of the cluster catalog, we cross-match the GMBCG clusters to X-ray clusters and maxBCG clusters. We also create a mock catalog based on DR7 data to test the completeness and purity of the catalog. The details of the catalog construction are covered in the following section. 


\subsection{Input catalog}

The Sloan Digital Sky Survey (SDSS) (York et al. 2000) is a multi-color digitized CCD imaging and spectroscopic sky survey, utilizing a dedicated 2.5-meter telescope at Apache Point Observatory, New Mexico. It has recently completed mapping over one quarter of the whole sky in ugriz filters. DR7 is a mark of the completion of the original goals of the SDSS and the end of the phase known as SDSS-II (Abazajian \& Sloan Digital Sky Survey 2008). It includes a total imaging area of 11663 square degrees with 357 million unique objects identified.

In this paper, we will mainly detect clusters on the so called Legacy Survey area, which "provided a uniform, well-calibrated map in ugriz of more than 7,500 square degrees of the North Galactic Cap, and three stripes in the South Galactic Cap totaling 740 square degrees" (Abazajian \& Sloan Digital Sky Survey 2008). We construct the input galaxy catalog from the CasJobs (http://casjobs.sdss.org/CasJobs/) PhotoPrimary view of the SDSS Catalog Archive Server with type set to 3 (galaxy) and $i$-band magnitude less than 21.0. In addition, we also apply the following flags to keep the catalog clean: SATURATED, SATUR_CENTER, BRIGHT, AMOMENT_MAXITER, AMOMENT_SHIFT and AMOMENT_FAINT. We download the photo- $z$ table and cross match the objects to the galaxy catalog to attach photo-zs to each galaxy we selected. In DR7, the photo- $z \mathrm{~s}$ in the photo- $z$ table are calculated based on a nearest neighbor polynomial algorithm (Abazajian \& Sloan Digital Sky Survey 2008).

In addition to the above selection requirements, we also throw away those galaxies with bad measurements (photometric errors in $g$ and $r$ band greater than 10 percent). In principle, we should search all galaxies as candidate BCGs. However, as BCG are well-known and form a subset of the total galaxy population, the list (and computational time) can be reduced. Based on Figure 3, we make cuts in color space as shown in the red 
regions of Figure 7. Additionally, each galaxy has a well-measured ellipticity through the SDSS data processing pipeline based on adaptive moments (Bernstein \& Jarvis 2002). We require the ellipticity in the $r$-band to be less than 0.7 for candidate BCGs. This ellipticity cut helps to remove edge on spiral galaxies which, when reddened by dust, often take on the colors of much higher redshift red sequence galaxies, and hence can appear as false projected BCGs. All these cuts keep $\sim 70 \%$ of the total galaxies in our candidate BCG search list, effectively eliminating only those with quite atypical colors and morphologies.

After the above procedures, we prepare an input catalog for our cluster finder. It is worth noting that we did not apply any star/galaxy separation procedures other than the ones generated by the standard DR7 pipeline. This is a relatively tolerant selection that may be contaminated by occasional bright stars that are not well separated from galaxies. As described earlier, we handle these stragglers by comparing the measured luminosity weighted clustering strength $\left(S_{\text {cluster }}^{\text {lum }}\right)$ with the non-luminosity weighted clustering strength $\left(S_{\text {cluster }}\right)$ to reject those bright stars.

\subsection{Richness Re-scaling}

In the redshift range $0.1 \sim 0.55$, only the $g-r$ or $r-i$ ridgeline colors are used, and the switch between them is determined by the photo- $z$ of the candidate BCG. Since we measure the richness by counting the number of galaxies falling within $2 \sigma$ of the ridgeline, the resulting richness from $g-r$ or $r-i$ are not directly comparable. In part this is due to a changing degree of background contamination as the ridgeline moves through color space (see Figure 14). Generally, the richness measured from $r-i$ is higher than that measured from $g-r$. To make the richnesses more consistent across the whole redshift range, we rescale those measured from $r-i$ color. Clearly, mass is the only true parameter with which we should relate the two different richness. Therefore, a complete resolution of this problem 


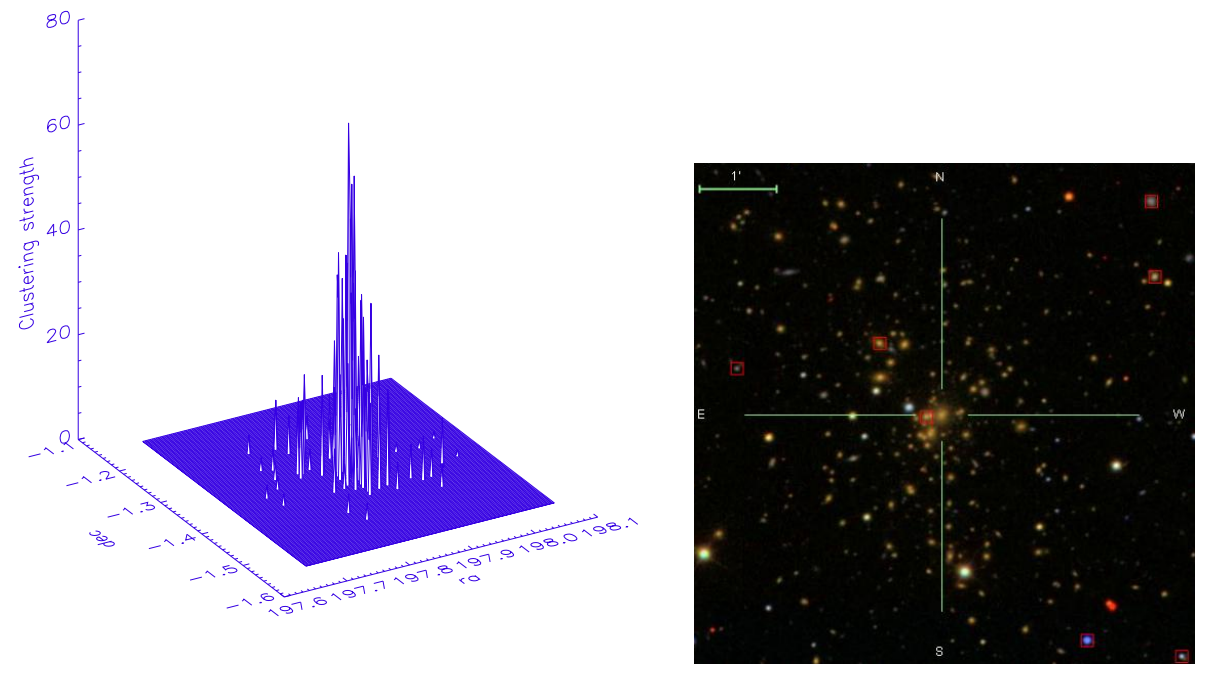

Fig. 6.- Left panel shows the clustering strength distribution around a galaxy cluster (Abell 1689). In this case, the BCG is the highest peak. Right panel: SDSS image of A1689.
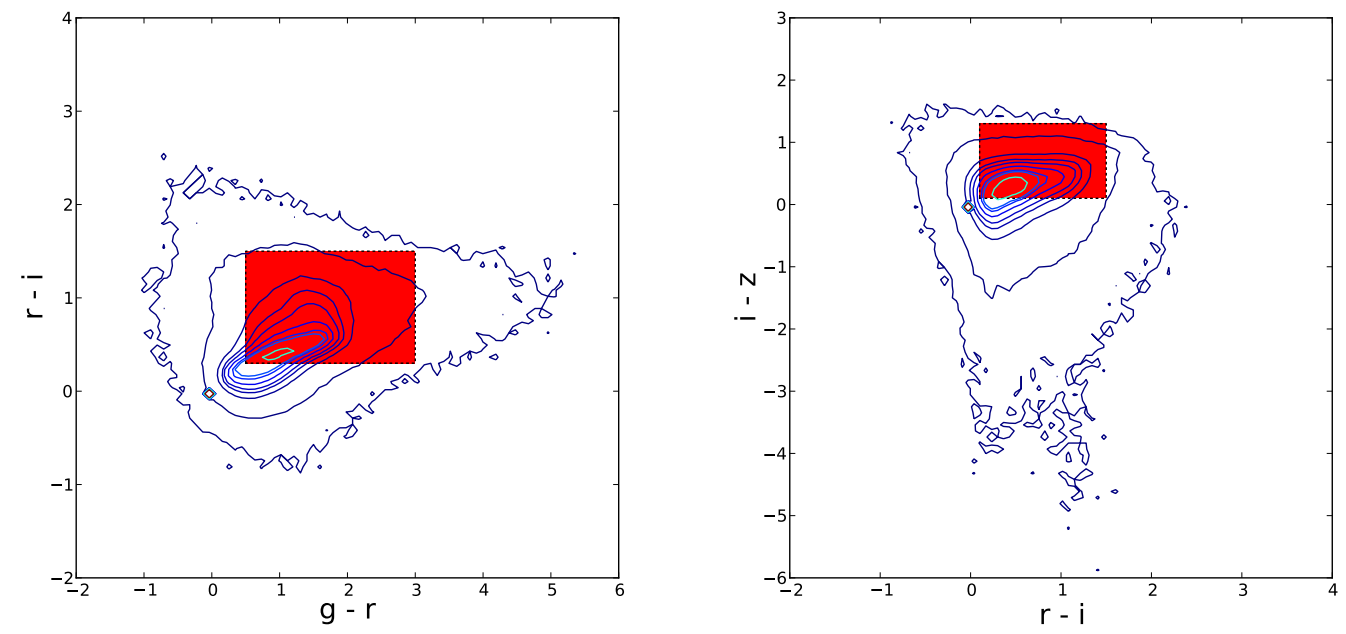

Fig. 7.- BCG preselection in color - color space for the SDSS DR7 data. Red regions indicate the area of $g-r$ vs. $r-i$ (left panel) and $r-i$ vs. $i-z$ (right panel) color-color space in which we preselect BCGs. This preselection keeps $\sim 70 \%$ of the total galaxies. 
requires a carefully mapping of the mass-richness relation for richness in both redshift ranges. However, for the moment, we settle for the simpler first order approach. That is, we require the statistical distribution of richness measured from $g-r$ color at redshift range $[0.41-0.43]$ and richness measured from $r-i$ color at redshift range $[0.43,0.45]$ to be the same since the true richness of the clusters in these narrow redshift ranges should vary only mildly. The scaling relation that matches the two distributions is not necessarily linear. To ensure the distribution to be the same, we match the richness at different percentile bins of the two distributions and re-scale them linearly in each bin. Then, we fit a polynomial to the scaling relation across all the bins to derive a "continuous" scaling relation. The richness from the $r-i$ color will be accordingly re-scaled by this relation. In Figure 8, we show the richness distribution before and after the re-scaling. Since the scaling relation is monotonously increasing, the scaled richness will not alter the cluster ranking based on the original richness in the $r-i$ region (it will affect the global ranking for sure). In a similar fashion, we also re-scale the weighted richness and the clustering strength. In the following, unless noted otherwise, the richness and clustering strength all refer to the rescaled values.

\subsubsection{Catalog Cleaning and Masking}

We apply the GMBCG algorithm to the input catalog and generate a full catalog of galaxy clusters for the SDSS DR7. We search clusters from redshift $0.05<z<0.60$, but only include in the final catalog the redshift range $0.1<z<0.55$ to reduce redshift range

edge effects. The luminosity weighted and non-luminosity weighted clustering strength (see above) are employed. For stars, the luminosity weighted clustering strength is much greater than its non-luminosity weighted counterpart. By hand scaning the corresponding images, we found the cuts as shown in Figure 4.2.1 are good for removing the contaminated stars.

In addition to the above cuts, we also mask out those clusters that are close to the 
brightest stars. We apply the bright star mask from the NYU VAGC (valued added galaxy catalog) release for SDSS DR7 (Blanton et al. 2005) and mask out all clusters that fall inside the bright star mask polygons.

\subsubsection{Catalog Facts}

Cleaning and masking trims the final catalog down to 380,000 clusters, which we will refer as full catalog. When we apply a richness cut $N_{\text {gals }}^{\text {scaled }} \geq 8$, we are left with about 55,000 rich clusters, which we release with this paper. We refer this as the "public catalog" and its sky coverage is shown in Figure 10. In Table 3, we list the tags in the public cluster catalog and their corresponding definitions. The redshift and richness distributions of the

clusters in the public catalog are shown in Figure 11. Images of example clusters at different redshifts are shown in Figure 12.

An inherent assumption in GMBCG is that the BCG's photo- $z$ should be determined much better than the rest of galaxies. We now test that assumption. In the public catalog, about 11,000 BCGs have spectroscopic redshift. In Figure 13, we show the performance of photo- $z$ for BCGs. The rms of the difference between BCG and photo- $z$ is $\sim 0.015$, which is almost the same as the photo- $z$ s from maxBCG clusters (Koester et al. 2007a), an indication that the assumption is secure.

\subsection{Bimodality in color Space}

As we have shown in previous sections, the apparent color distribution around a cluster generally shows bi-modality. However, there are situations where the cluster is so big that its members completely dominate the field within the aperture we impose; in this case, the color distribution may be uni-modal. In our implementation of the GMBCG algorithm, 
Table 3: Tags in the cluster catalog

\begin{tabular}{ll}
\hline \hline Tag Name in catalog & Definition \\
\hline OBJID & Unique ID of each galaxy in SDSS DR7 \\
RA & Right Ascention \\
DEC & Declination \\
PHOTO- $Z$ & photo- $z$ from the photo- $z$ table in DR7 \\
PHOTO-Z_ERR & Errors of photo- $z$ \\
SPZ & Spectroscopic redshift \\
GMR & $g-r$ color ${ }^{a}$ \\
GMR_ERR & Error of $g-r$ color \\
RMI & $r-i$ color \\
RMI_ERR & Error of $r-i$ color \\
MODEL_MAG & Dust extinction corrected model magnitude ${ }^{b}$ \\
MODEL_MAG_ERR & Error of model magnitude \\
S_CLUSTER & Clustering strength, $S_{\text {cluster }}$ \\
GM_SCALED_NGALS & Number of member galaxies inside GM_SCALEDR from BCG \\
GM_NGALS_WEIGHTED & Weighted richness. \\
\hline
\end{tabular}

${ }^{a}$ All colors are calculated using model magnitude

${ }^{b}$ For details, see http://www.sdss.org/DR7/algorithms/photometry.html 
we also consider this situation as a potential cluster as long as the width of the dominant uni-modal distribution is narrow enough (width $<0.16$ ).

In the case of a bimodal color distribution, the separation between the two Gaussian components will vary as redshift changes, leading to different degrees of overlap. This overlap of the two Gaussian components measures the fraction of projected galaxies when we impose the color cuts on the red sequence galaxies. Therefore, the richness for the clusters should be appropriately weighted to account for the projection. In Figure 14, we show the color distribution of clusters at different redshifts. From the plot, the $2 \sigma(\sigma$ is the standard deviation of the Gaussian component corresponding to red sequence) cut we imposed for selecting red sequence members coincides with point at which the likelihood of red sequence galaxy becomes equal to that of background/blue galaxies.

This information is important for getting consistent richness estimates across the redshift range. The $2 \sigma$ clip we use to select member galaxies will lead to different levels of background galaxy contamination at different redshifts. The weighted richness introduced in $\S 3.4 .4$ takes this overlap into account automatically and thus is a better richness estimator than the direct cluster member counts based on the top-hat $2 \sigma$ color cuts. However, the weighted richness is not always better than the direct number counts. There are two cases that demand caution when the weighted richness is used. In first case there is only one Gaussian component, which does not permit a weighted richness. The second case is that there are situations where the relative weight estimates from the ECGMM is not reliable, e.g. very small, leading to a very small weighted richness. In this case, we recommend the direct richness counts, i.e. $N_{\text {gal }}^{\text {scale }}$. 


\section{Evaluating the catalog}

Any cluster finding algorithm can be evaluated by two simple criteria: completeness and purity. Completeness quantifies whether the cluster finder can find all true clusters, while purity quantifies whether the clusters found by the cluster finder are real clusters. However, calculating the completeness and purity requires that we know in advance what is a true cluster. Ideally, the true cluster here should correspond to a dark matter halo. This issue can only be completely resolved when we have a high resolution simulation that can properly reflect the galaxies' colors as well as their interaction with dark matter halos. However, creating a realistic galaxy catalog from the N-body simulation has proven to be very challenging, complicated by various factors such as unknown physics processes, limited resolution of simulation, unknown behaviour of galaxies at high redshift, and other complications that affect the evolution of galaxy colors and distribution. Therefore, in practice, we need to slightly change the definition of true cluster to certain model clusters we defined in terms of observational features.

In this section, we introduce a simple but realistic mock catalog to test our cluster finder. The result can tell us the purity and completeness of our cluster catalog with respect to the model clusters we put in. In addition, as a check of completeness of the cluster catalog, we also cross match our clusters to X-ray clusters and clusters from maxBCG catalog. Considering uncertainties in cluster richness measurement, we will use the full catalog in this section to accommodate the richness variances.

\subsection{Mock catalog}

Inserting model clusters into a realistic background is a widely used method to create mock catalogs for evaluating cluster finding algorithms (Diaferio et al. 1999; Adami et al. 
2000; Postman et al. 2002; Kim et al. 2002; Goto et al. 2002; Koester et al. 2007a). In practice, there are different schemes to make the mock catalog as realistic as possible. In this paper, we develop a Monte Carlo scheme that is similar to those used in (Goto et al. 2002; Koester et al. 2007a), but with additional features. We construct mock catalogs in four steps:

1. The background galaxy distribution: To make a realistic background, we consider 25 stripes from our input galaxy catalogs from SDSS DR7. We remove the rich clusters (richness greater than 20 in our cluster catalog, about $4 \%$ of the total galaxy in the input catalog) and shuffle the remaining galaxies' positions (ra/dec), while keeping their colors and other properties unchanged, creating a 'base' catalog.

2. Model cluster selection: We select 49 rich clusters whose redshift ranges from 0.1 to 0.55 from our cluster catalog. About $60 \%$ of these clusters have a match with known x-ray clusters (see §5.4) and all of them have been visually checked to be very rich. Each cluster has a BCG and about 30-100 member galaxies brighter than $0.4 L^{*}$.

3. Model mock clusters re-sampling: Pick a BCG randomly from the 49 model clusters and then select a fixed number of member galaxies from the corresponding model cluster's members. The fixed number is randomly chosen from [10, 15, 20, 25, 30, 35, 40, 45, 50]. The relative positions, colors and luminosities of these galaxies all remain unchanged with respect to BCG. In this way, we can generate a re-sampled model cluster of a given richness.

4. Putting re-sampled model clusters into base catalog: For every stripe of the base catalog, we select 500 re-sampled model clusters (roughly the number of clusters removed in step 1) and put them into the background galaxy catalog so that their corresponding BCGs replace 500 randomly chosen galaxies in the base catalog. Then, 
we will have a Monte Carlo catalog that are based on the real photometry of the SDSS DR7 data.

By construction, the Monte Carlo catalog is based on actual SDSS photometry, and produces a mock catalog with reasonably realistic background galaxies.

\subsection{Completeness and Purity}

To test the completeness and purity of our cluster finder, we run it on the mock catalog created above. Then, we cross match the detected clusters and the model clusters using a simple cylinder matching, i.e. searching in a cylinder of $R_{\text {scale }}$ in radius and \pm 0.05 in redshift. When we test the completeness, we firstly sort the model cluster list by the cluster richness and then match the detected clusters to them through the cylinder match. While we test the purity, we sort the detected cluster list by their richness and then match the model clusters to them via the cylinder match. In both cases, we will consider only those unique and exclusive matches, meaning that a model cluster will not be used any more once it is matched to a detected cluster for purity test and a detected cluster will not be used any more once it is matched to a model cluster for the completeness test. If more than one cluster falls in the cylinder, we choose the richest one. After doing the matching, at a given redshift bin and above a given $N_{\text {gal }}$, the completeness and purity can then be defined as

$$
\begin{gathered}
\text { completeness }=\frac{N_{\text {model }}^{\text {match }}\left(z, N_{\text {gal }}\right)}{N_{\text {model }}\left(z, N_{\text {gal }}\right)} \\
\text { purity }=\frac{N_{\text {found }}^{\text {match }}\left(z, N_{\text {gal }}^{\text {scaled }}\right)}{N_{\text {found }}\left(z, N_{\text {gal }}^{\text {scaled }}\right)}
\end{gathered}
$$

where $N_{\text {model }}^{\text {match }}\left(z, N_{\text {gal }}\right)$ denote the number of model clusters that are matched to the found clusters by,$N_{\text {model }}\left(z, N_{\text {gal }}\right)$ is the total number of model clusters, $N_{\text {found }}^{\text {match }}\left(z, N_{\text {gal }}^{\text {scaled }}\right)$ is the number of found clusters that are matched to model clusters and $N_{\text {found }}\left(z, N_{\text {gal }}^{\text {scaled }}\right)$ is the 
total number of found clusters. The results of the completeness and purity are plotted in Figure 15. The plot show that the GMBCG algorithm can yield a highly complete and pure cluster catalog.

\subsection{Cross-Matching of GMBCG clusters to MaxBCG Clusters}

As a further test of the completeness of the GMBCG catalog, we make a comparison to the maxBCG catalog (Koester et al. 2007a). The maxBCG catalog consists of 13,823 clusters in the redshift range $0.1<z<0.3$ with a lower threshold on richness set at $N_{200}=10$. It is derived from DR5 of the Sloan Digital Sky Survey and covers a slightly smaller area than the new GMBCG catalog.

Several complications arise in the process of performing cluster-to-cluster matches between catalogs, namely redshift uncertainties, centring differences between the two algorithms, and scatter in the richness measurements. Although many similarities exist between the maxBCG and GMBCG algorithms, it is not always the case that they choose the same central galaxy for a given cluster. When matching clusters, a careful cut must be made in the two-dimensional physical separation in order to allow for this centring ambiguity, while at the same time minimizing matches due to random projection. Uncertainty in the photometric redshifts can yield a similar problem along the line of sight; a cut in $\Delta z=\left|z_{\max B C G}-z_{G M B C G}\right|$ must be made to accommodate these errors. Finally, the richness measurements themselves have large scatter, i.e. clusters that appear in one catalog may have richness values below the richness threshold of the counterpart catalog, rendering them unavailable to match. These problems ultimately will determine a reasonable matching scheme to that can be used to quantify the agreement between the GMBCG catalog and the maxBCG catalog. We now consider these effects. 
The uncertainty in redshift estimates for maxBCG clusters is $\sigma_{z} \sim 0.015$ (Koester et al. 2007a). In the GMBCG catalog, the uncertainty of the photo- $z$ s at redshift below 0.3 is $\sim 0.016$ (Figure13). Therefore, a redshift difference of $\sim 0.05$ between the two catalogs is an appropriate selection window for matching. As for the radial separation, given the fact that the maxBCG clusters are percolated within a separation of $R 200 \sim 1.0-2.0 \mathrm{Mpc}$ (Koester et al. 2007b), a radial separation of $\sim 2.0 \mathrm{Mpc}$ is appropriate for our matching search. Generally speaking, the smaller the matching separation, the higher the probability of real matches. Also, the lower the richness of the maxBCG cluster, the less likely they are true clusters. Therefore, we will represent our matching with respect to both the separation and the richness of maxBCG clusters.

We hold the maxBCG clusters as target and match the clusters from our full GMBCG catalogs to them. In other words, it is essentially a completeness test of the GMBCG catalog. We then execute the cylindrical matching algorithm described above. The matching yields that 13,374 out of $13,823(\sim 96.8 \%)$ clusters in maxBCG catalog have a match in the GMBCG catalog. Those non-matched clusters are mostly at low richness end, which is mainly due to the low end cuts placed on the catalog. There are also 8,818 of the 13,374 matched clusters $(\sim 65.9 \%)$ that have identical BCGs in both catalogs. In the left panel of Figure 16, we show the matching fraction of the GMBCG clusters to maxBCG clusters at different maxBCG richness and separation. As a comparison, we create a control catalog of the same size as the GMBCG catalog, but with the ra and dec randomized. The matching results of this control catalog to maxBCG clusters are shown in the right panel of Figure 16. 


\subsection{Cross-Matching of GMBCG to ROSAT X-ray Clusters}

Optical identification of peaks in the galaxy distribution represents only one of many methods used to find clusters. Other observables employed in cluster detection include thermal emission of x-rays from the hot intra-cluster medium, weak-lensing distortion of background sources, and the Sunyaev-Zeldovich effect of hot gas on the cosmic microwave background. Each method has certain advantages and disadvantages. Each also provides a

distinct proxy for the mass of a cluster, which can be used to probe cosmological constraints. It is important that our cluster finding algorithm be able to detect those clusters found by alternative means. X-ray cluster catalogs are the most appealing candidate for exploring this question. Numerous x-ray catalogs exist with large sky coverage overlapping the DR7 survey area. Follow up optical examination is frequently performed on these catalogs to confirm their identity as clusters and is required to obtain redshifts.

Matching complications likewise arise when comparing to X-ray catalogs. It is not always the case that the BCG lies exactly on the X-ray peak. There also exists significant scatter in the x-ray luminosity-richness relation (Rykoff et al. 2008). Furthermore, the DR7 catalog contains clusters down to a richness threshold much lower then current x-ray catalogs can detect. The main goal of this subsection is to test the extent to which our algorithm is able to identify the most luminous x-ray clusters.

We compare the DR7 catalog to three x-ray identified cluster catalogs: NORAS (Böhringer et al. 2000), REFLEX (Böhringer et al. 2004) and $400 \operatorname{deg}^{2}$ (Burenin et al. 2007). NORAS and REFLEX consist of clusters identified from extended sources on the ROSAT all-sky survey x-ray maps. Together they cover the northern and southern galactic caps and are flux limited at $3 \times 10^{-12} \mathrm{ergs} \mathrm{s}^{-1} \mathrm{~cm}^{-2}$ in the $0.1-2.4 \mathrm{KeV}$ energy band. The $400 \mathrm{deg}^{2}$ catalog is composed of serendipitous clusters found in the high galactic latitude ROSAT pointings. It is flux limited at $1.4 \mathrm{~s}^{-1} \mathrm{~cm}^{-2}$ in the $0.5-2.0 \mathrm{KeV}$ energy band. 
Sources from all three catalogs have been confirmed as clusters through follow up optical identification. Combining these catalogs yields 229 unique clusters in the survey area spanned by DR7.

A cylindrical search is performed on the combined x-ray catalogs in order to determine if these clusters were found by the GMBCG algorithm, effectively a completeness test of GMBCG. We consider two clusters a match if they have a physical separation in the projected plane sep $<2.0 \mathrm{Mpc}$ and a redshift difference $\left|z_{\text {xray }}-z_{\text {photo }}\right|<0.05$. By this criteria, 227 out of 229 X-ray clusters are matched with at least one GMBCG cluster. In Figure 17, we show the images of the two non-matched X-Ray clusters. For the GMBCG clusters, the higher the richness and clustering strength, the more likely they are true clusters. Recalling that the shorter the matching separation, the higher probability of a true match, in Figure 18 we show the contour plots of the matching ratio in terms of separation and cluster richness and clustering strength. The results confirm high completeness: we can reliably recover about $90 \%$ of the X-Ray clusters with separation less than $0.5 \mathrm{Mpc}$ and richness greater than 8.)

\section{Discussion}

In this paper, we present a new cluster finding algorithm, GMBCG, and publish the largest ever optical cluster catalog, with more than 55,000 rich clusters. GMBCG identifies galaxy clusters by detecting the BCG plus red sequence feature that exists only in galaxy clusters and is not possessed by field galaxies. This feature provides a powerful means for detection of galaxy clusters with minimal line-of-sight projection contamination. The effectiveness of this algorithm is based on the assumption that a BCG plus red sequence feature is "universal" among galaxy clusters. Though this feature is preserved in almost all

clusters known to us, we cannot exclude the possibility that there are some clusters that do 
not have this feature. In particular, this is more likely at very high redshift where clusters are forming. However, even if such "blue" clusters exist, it will be very challenging to detect them using photometric data in optical bands in a consistent way across a range of richness values.

The GMBCG algorithm uses the BCG's photo- $z$ to determine the metric aperture size, and uses the red sequence color to select member galaxies. It separates the process of getting photo- $z$ s and detecting clusters. This differentiates it from matched filter algorithms (including maxBCG). For the SDSS data the precision of the photo-zs for the BCGs from the machine learning algorithms are within a factor of 2 of the photo-z's from maxBCG, which means there is not a serious disadvantage in this choice. Using existing photo- $z \mathrm{~s}$ significantly boosts the computation efficiency. GMBCG can produce a cluster catalog for the full SDSS DR7 within 23 hours on a DELL computer with a single quad core CPU and 8G RAM. As long as the photo- $z$ is not catastrophically bad, GMBCG can detect the BCG plus red sequence feature of clusters; though richness measurements may be affected by imprecise redshift estimates.

It is worth noting that for cosmological application, we generally want to know the best mass proxy. Recent work has shown that weighted richnesses are among the best optical mass proxies, rather than the direct counts of member galaxies (Rozo et al. 2008). However, this does not mean that we should abandon the direct member galaxy count and identification. On the contrary, it will be very interesting to have the member galaxies explicitly determined for cluster science, i.e., the formation and evolution of clusters.

Though GMBCG works very well for the current SDSS DR7 data, there is still room for improvement, especially for deeper data. For example, GMBCG does not work well for very low richness clusters, say richness less than 4 for SDSS DR7 data. This is mainly because GMM/ECGMM will not reliably detect the red sequence at such low richness. 
GMBCG relies on the good photo-zs for BCGs, which may be risky at very high redshift where photo- $z$ precision is not guaranteed. The current GMBCG implementation relies on the photo- $z$ to decide the color to search for the red sequence. This is not a serious issue for the current data set, but will be preferable to perform a more comprehensive analysis on color space spanned by all colors. These are beyond the scope of this paper and additional improvements are left to future work on deeper data, such as SDSS co-added data and the incoming Dark Energy Survey data (The Dark Energy Survey Collaboration 2005). 

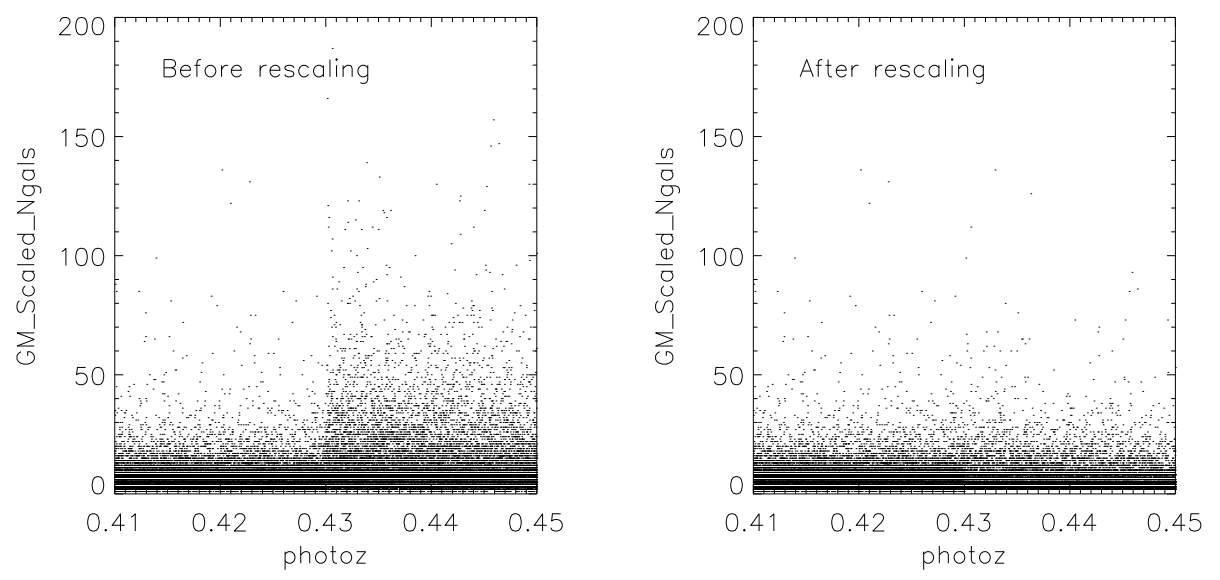

Fig. 8.- Richness $\left(N_{\text {gal }}^{\text {scaled }}\right)$ before and after the re-scaling. This demonstrates that rescaling removes much of the difference in richness measurements between the g-r and $r-i$ bands. 


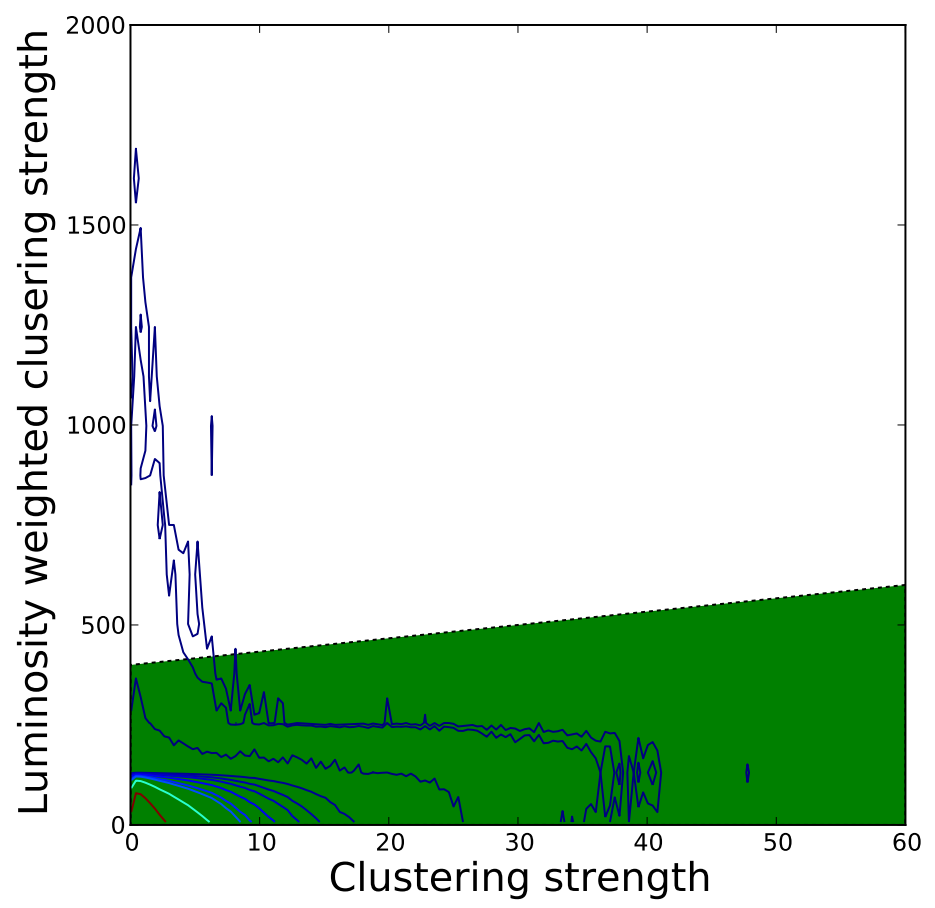

Fig. 9.- Density contour of BCGs in the space of luminosity weighted and non-luminosity weighted clustering strength. Blue contours show the results for all candidate BCGs. The green region shows cuts applied to candidate BCGs, as described in $\S 4.2 .1$, which removes bright stars that pass the star/galaxy separation in the SDSS data processing pipeline. 


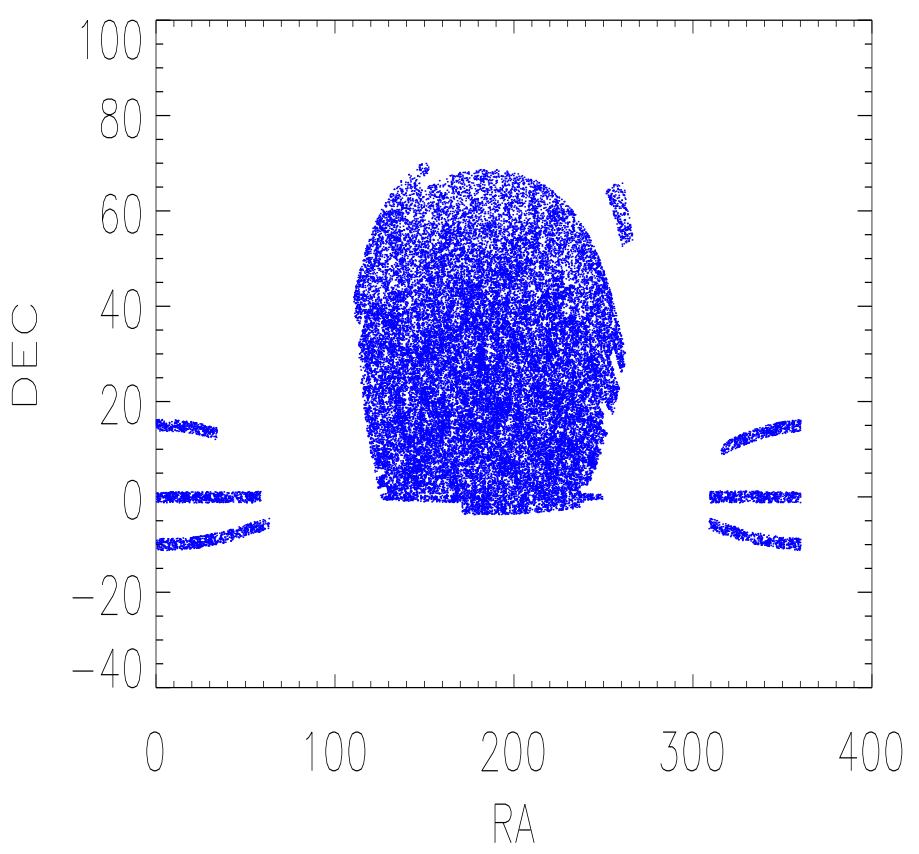

Fig. 10.- Sky coverage in the GMBCG public catalog based on SDSS DR7. Each point shows the position of one cluster on the sky. 

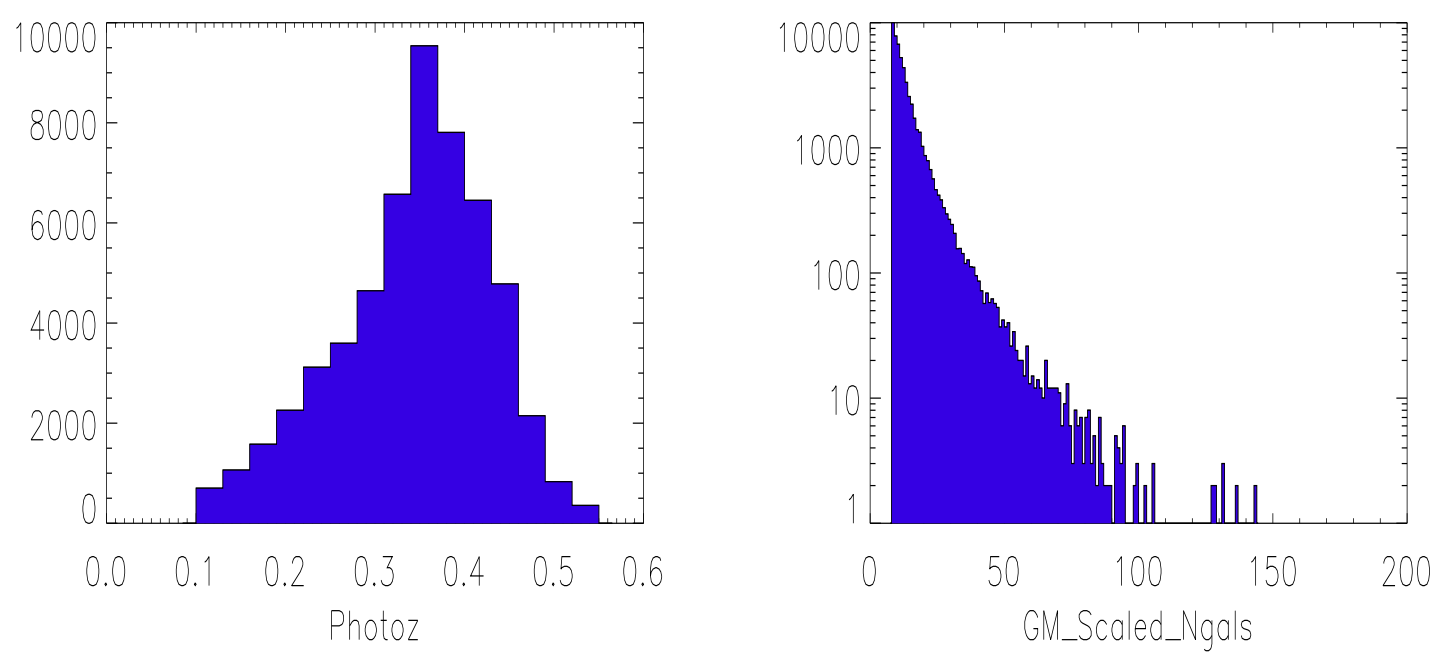

Fig. 11.- Redshift and richness distribution of GMBCG clusters in the public catalog. Left panel shows the redshift distribution of clusters, cut at $0.1<$ photo $-z<0.55$. Right panel shows the scaled richness distribution, GM_scaled_Ngals, for clusters with GM_scaled_Ngals $>8$. 

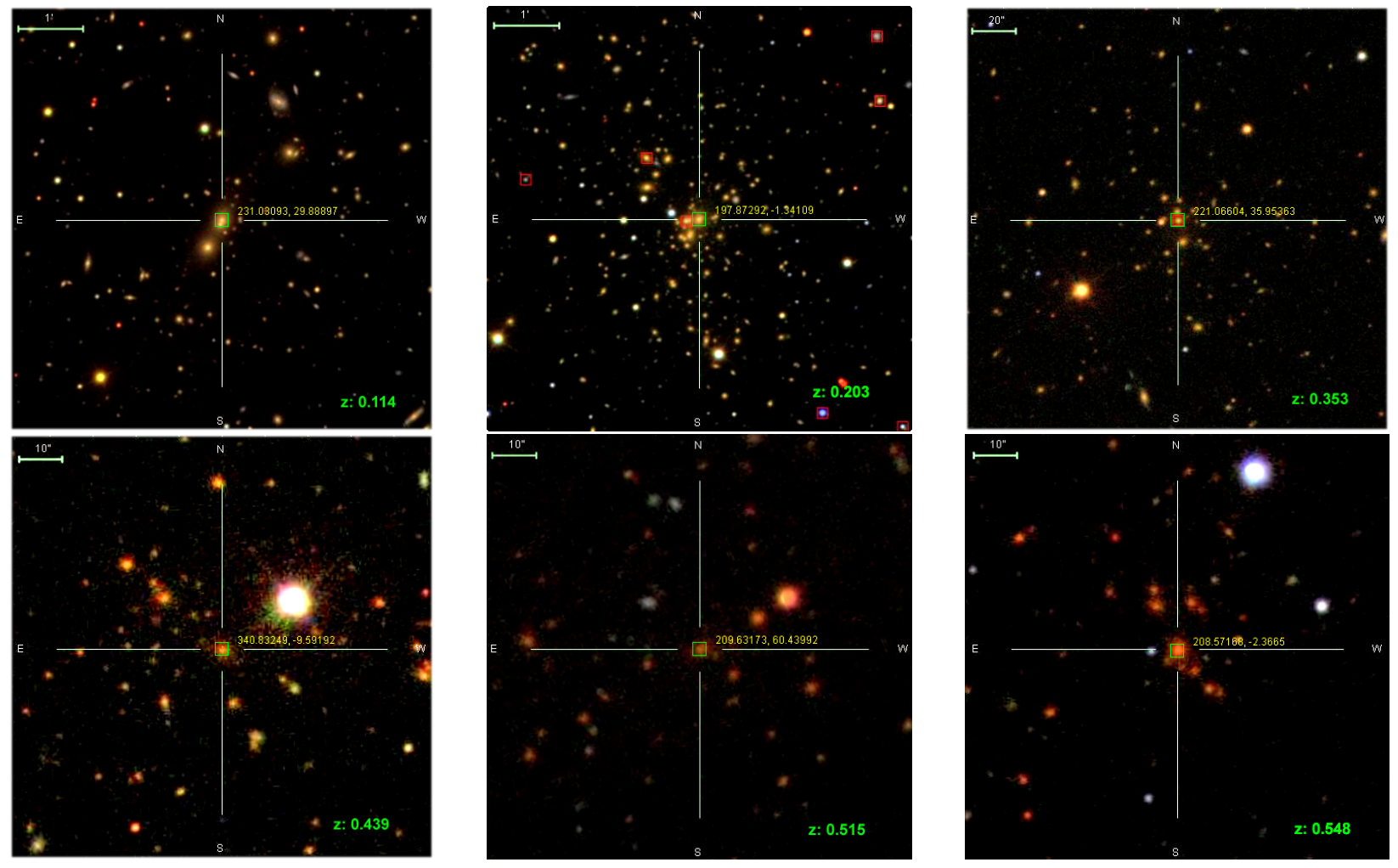

Fig. 12.- Sample cluster images from SDSS DR7 cluster catalog. The BCG spectroscopic redshift is given in green.
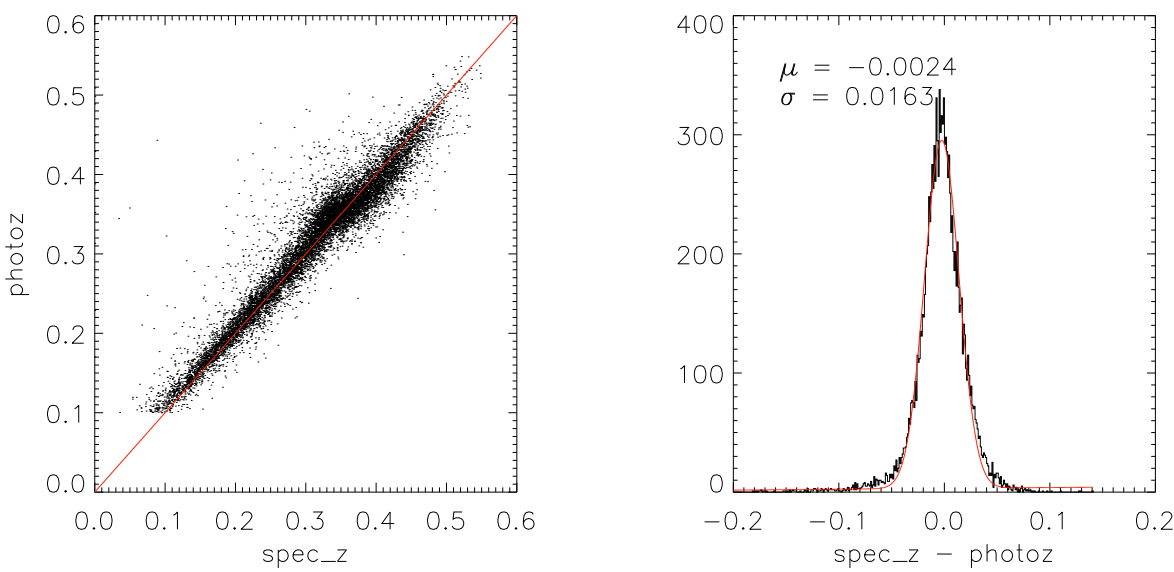

Fig. 13. - The difference between photo-z and spec-z for the BCGs in the public catalog. 

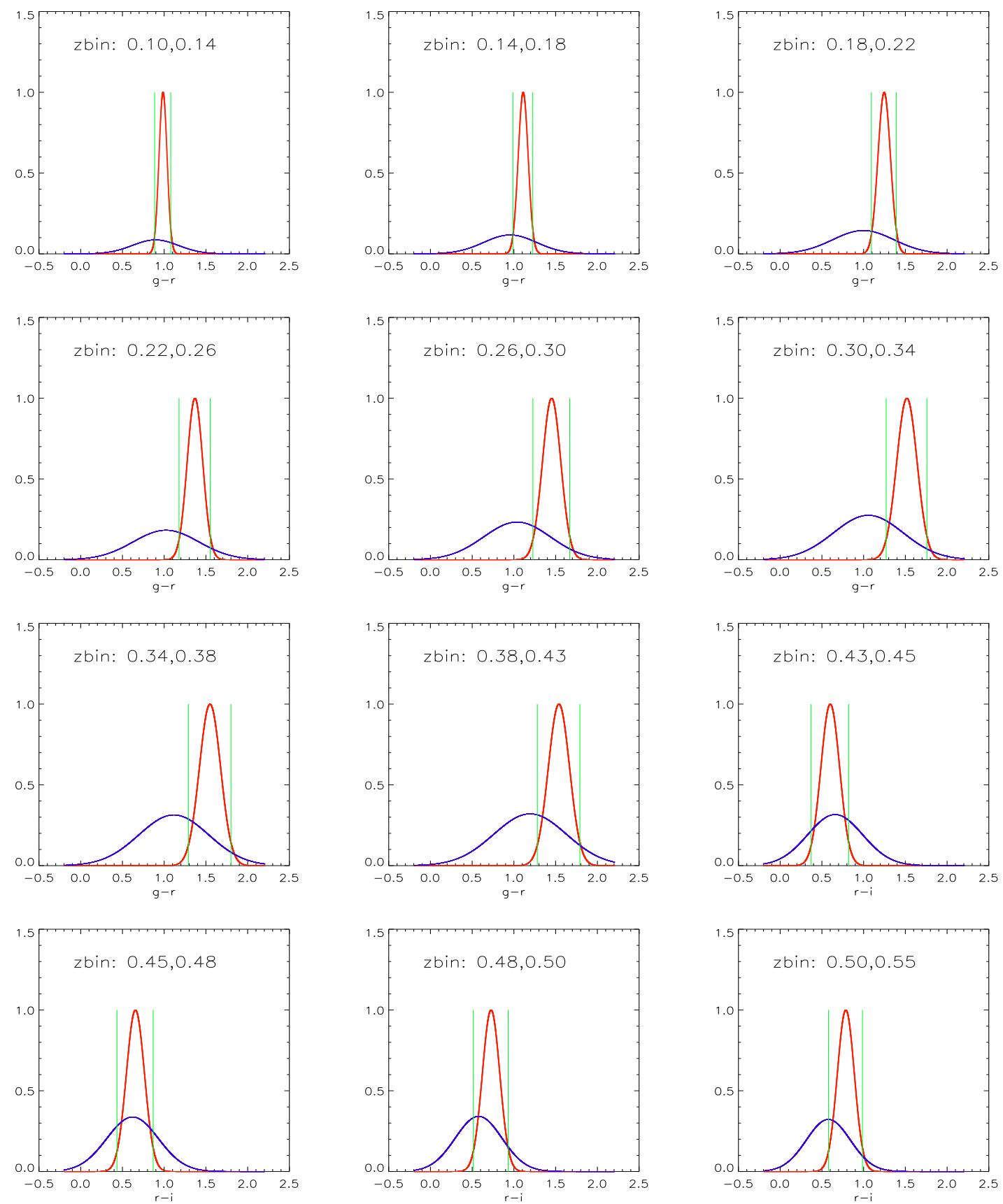

Fig. 14. - The bimodal distribution of red sequence galaxy colors and background/blue galaxies. The results are based on the average results of clusters falling in each redshift bin as indicated in the plots. The green vertical lines are the $2 \sigma$ clip of the red sequence peak. 


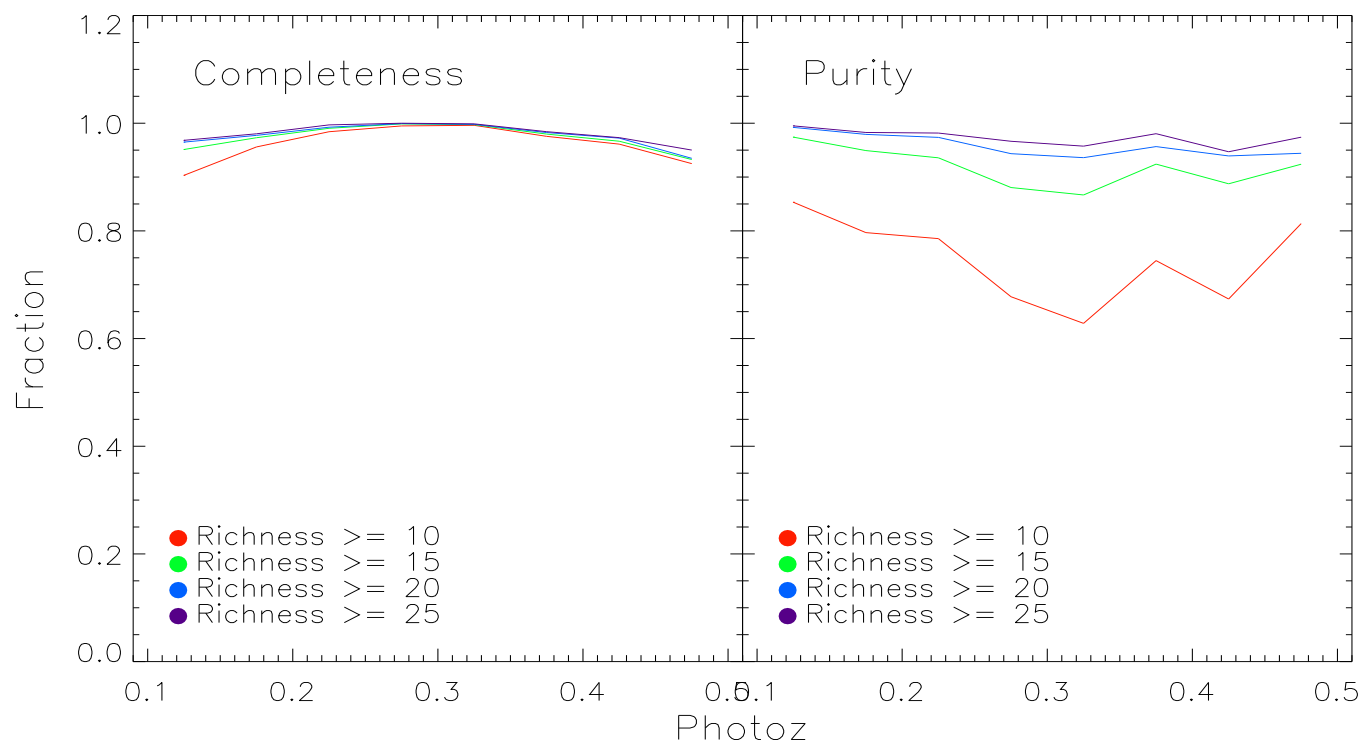

Fig. 15. - The completeness and purity of the GMBCG catalog based on the Monte Carlo catalog. In the completeness plot, $N_{g a l}$ is the number of member galaxies of our input model clusters. In the purity plot, $N_{\text {gal }}^{\text {scaled }}$ is the number of member galaxies measured by the cluster finder. Since there are residual red galaxies in the catalog before we put clusters in, the measured cluster richness $\left(N_{\text {gal }}^{\text {scaled }}\right)$ is generally higher than our input richness 

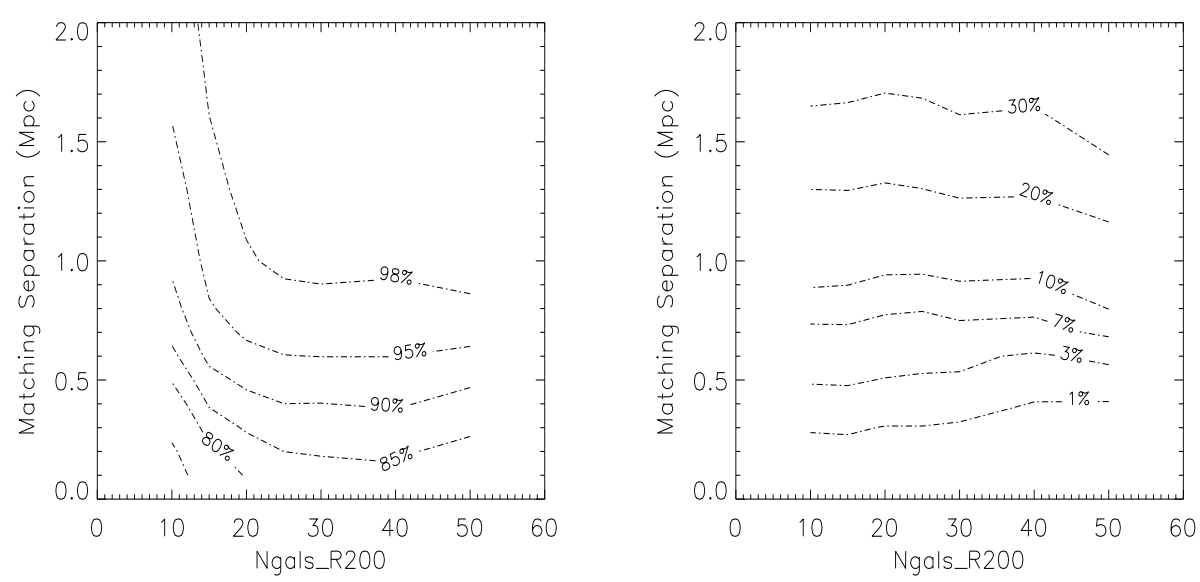

Fig. 16. - Left panel is the contour of matching fraction of the maxBCG clusters to the GMBCG clusters as a function of richness (Ngals_R200) and separations. In the right panel, we show the matching results from the control catalog of random positions.
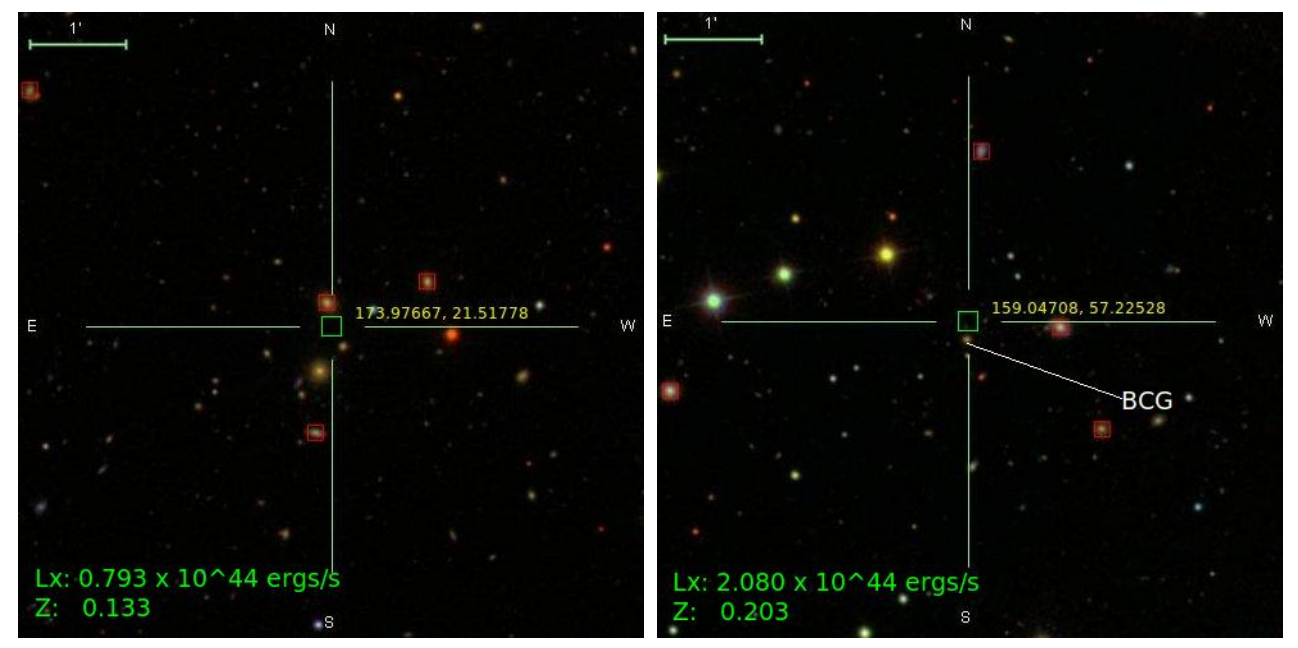

Fig. 17.- Two non-matched X-Ray clusters. The cluster on right panel actually has a BCG identified in the GMBCG catalog, but it is not recorded as a match because of the photo- $z$ of the BCG is assigned as 0.549, falling outside of our redshift matching envelope. 

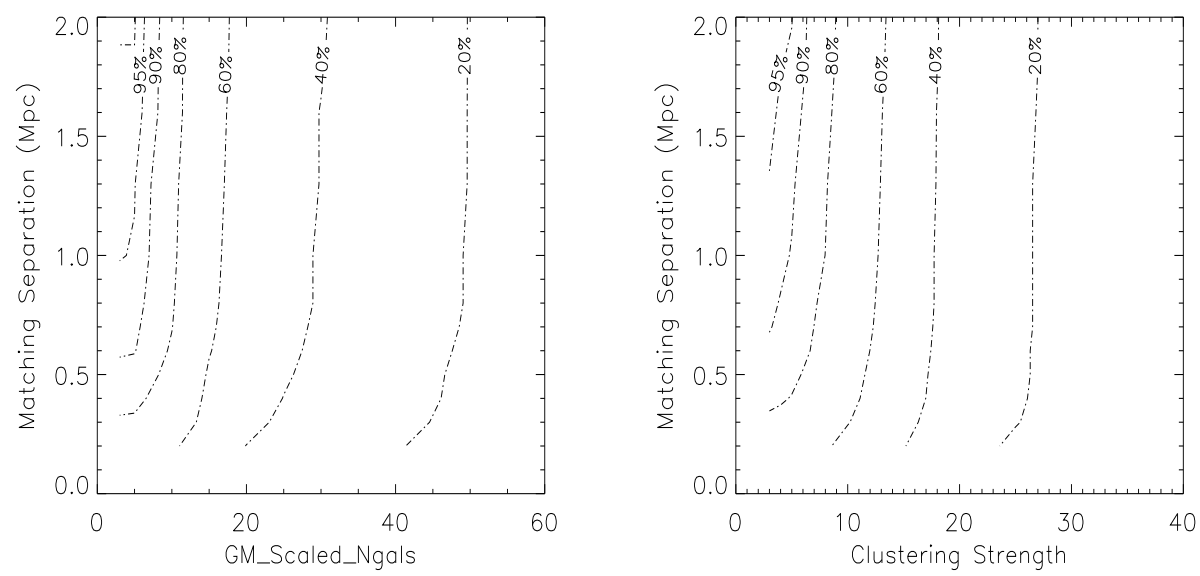

Fig. 18.- Contour of the matching ratio to ROSAT clusters for different separation and richness/clustering strength. The percentages are calculated by the ratio of the matched clusters with matching separation less than the point on the contour while richness/clustering strength greater than the point on the contour. 


\section{REFERENCES}

Abazajian, K., \& Sloan Digital Sky Survey, f. t. 2008, ArXiv e-prints

Abell, G. O. 1957, The distribution of rich clusters of galaxies. A catalogue of 2712 rich clusters found on the National Geographic Society Palomar Observatory Sky Survey (Chicago: Univiersity of Chicago Press, 1957)

Adami, C., Ulmer, M. P., Romer, A. K., Nichol, R. C., Holden, B. P., \& Pildis, R. A. 2000, ApJS, 131, 391

Akaike, H. 1974, Automatic Control, IEEE Transactions on, 19, 716

Annis, J., et al. 1999, in Bulletin of the American Astronomical Society, Vol. 31, Bulletin of the American Astronomical Society, 1391-+

Barger, A. J., et al. 1998, ApJ, 501, 522

Barrientos, L. F. 1999, PhD thesis, AA(UNIVERSITY OF TORONTO (CANADA))

Bartelmann, M. 1996, A\&A, 313, 697

Berlind, A. A., et al. 2006, ApJS, 167, 1

Bernstein, G. M., \& Jarvis, M. 2002, AJ, 123, 583

Blakeslee, J. P., et al. 2003, ApJ, 596, L143

—. 2006, ApJ, 644, 30

Blanton, M. R., et al. 2005, AJ, 129, 2562

Böhringer, H., et al. 2004, A\&A, 425, 367

-. 2000, ApJS, 129, 435 
Botzler, C. S., Snigula, J., Bender, R., \& Hopp, U. 2004, MNRAS, 349, 425

Bower, R. G., Lucey, J. R., \& Ellis, R. S. 1992, MNRAS, 254, 601

Burenin, R. A., Vikhlinin, A., Hornstrup, A., Ebeling, H., Quintana, H., \& Mescheryakov, A. 2007, ApJS, 172, 561

Couch, W. J., Ellis, R. S., MacLaren, I., \& Malin, D. F. 1991, MNRAS, 249, 606

Davis, M., Efstathiou, G., Frenk, C. S., \& White, S. D. M. 1985, ApJ, 292, 371

De Lucia, G., et al. 2007, MNRAS, 374, 809

Diaferio, A., Kauffmann, G., Colberg, J. M., \& White, S. D. M. 1999, MNRAS, 307, 537

Dong, F., Pierpaoli, E., Gunn, J. E., \& Wechsler, R. H. 2008, ApJ, 676, 868

Efstathiou, G., Frenk, C. S., White, S. D. M., \& Davis, M. 1988, MNRAS, 235, 715

Eisenhardt, P. R., et al. 2005, in Bulletin of the American Astronomical Society, Vol. 37, Bulletin of the American Astronomical Society, 1344-+

Eisenstein, D. J., et al. 2005, ApJ, 633, 560

Evrard, A. E. 1989, ApJ, 341, L71

Gal, R. R. 2006, ArXiv Astrophysics e-prints

Gal, R. R., de Carvalho, R. R., Lopes, P. A. A., Djorgovski, S. G., Brunner, R. J., Mahabal, A., \& Odewahn, S. C. 2003, AJ, 125, 2064

Gal, R. R., de Carvalho, R. R., Odewahn, S. C., Djorgovski, S. G., \& Margoniner, V. E. 2000, AJ, 119, 12 
Gerdes, D. W., Sypniewski, A. J., McKay, T. A., Hao, J., Weis, M. R., Wechsler, R. H., \& Busha, M. T. 2009, ArXiv e-prints

Gladders, M. D., Lopez-Cruz, O., Yee, H. K. C., \& Kodama, T. 1998, ApJ, 501, 571

Gladders, M. D., \& Yee, H. K. C. 2000, AJ, 120, 2148

-. 2005a, ApJS, 157, 1

—. 2005b, ApJS, 157, 1

Gladders, M. D., Yee, H. K. C., Majumdar, S., Barrientos, L. F., Hoekstra, H., Hall, P. B., \& Infante, L. 2007, ApJ, 655, 128

Goto, T., et al. 2002, AJ, 123, 1807

Hansen, S. M., Sheldon, E. S., Wechsler, R. H., \& Koester, B. P. 2007, ArXiv e-prints, 710

Hao, J., et al. 2009, ApJ, 702, 745

Hu, W. 2003, Phys. Rev. D, 67, 081304

Huchra, J. P., \& Geller, M. J. 1982, ApJ, 257, 423

Johnston, D. E., et al. 2007, ArXiv e-prints, 709

Kepner, J., Fan, X., Bahcall, N., Gunn, J., Lupton, R., \& Xu, G. 1999, ApJ, 517, 78

Kim, R. S. J., et al. 2002, AJ, 123, 20

Koester, B. P., et al. 2007a, ApJ, 660, 239

-. 2007b, ApJ, 660, 221

Li, I. H., \& Yee, H. K. C. 2008, AJ, 135, 809 
Lidman, C. E., \& Peterson, B. A. 1996, AJ, 112, 2454

Lima, M., \& Hu, W. 2004, Phys. Rev. D, 70, 043504

—. 2005, Phys. Rev. D, 72, 043006

Lopes, P. A. A., de Carvalho, R. R., Gal, R. R., Djorgovski, S. G., Odewahn, S. C., Mahabal, A. A., \& Brunner, R. J. 2004, AJ, 128, 1017

López-Cruz, O., Barkhouse, W. A., \& Yee, H. K. C. 2004, ApJ, 614, 679

Majumdar, S., \& Mohr, J. J. 2004, ApJ, 613, 41

Mei, S., et al. 2009, ApJ, 690, 42

Miller, C. J., et al. 2005, AJ, 130, 968

Mullis, C. R., Rosati, P., Lamer, G., Böhringer, H., Schwope, A., Schuecker, P., \& Fassbender, R. 2005, ApJ, 623, L85

Navarro, J. F., Frenk, C. S., \& White, S. D. M. 1997, ApJ, 490, 493

Oukbir, J., \& Blanchard, A. 1992, A\&A, 262, L21

Oyaizu, H., Lima, M., Cunha, C. E., Lin, H., Frieman, J., \& Sheldon, E. S. 2007, ArXiv e-prints, 708

Papovich, C., et al. 2010, ApJ, 716, 1503

Perlmutter, S., et al. 1999, Astrophys. J., 517, 565

Postman, M., Lauer, T. R., Oegerle, W., \& Donahue, M. 2002, ApJ, 579, 93

Postman, M., Lubin, L. M., Gunn, J. E., Oke, J. B., Hoessel, J. G., Schneider, D. P., \& Christensen, J. A. 1996, AJ, 111, 615 
Ramella, M., Geller, M. J., Pisani, A., \& da Costa, L. N. 2002, AJ, 123, 2976

Riess, A. G., et al. 1998, Astron. J., 116, 1009

Rozo, E., et al. 2008, ArXiv e-prints

Rozo, E., Wechsler, R. H., Koester, B. P., Evrard, A. E., \& McKay, T. A. 2007a, ArXiv Astrophysics e-prints

Rozo, E., et al. 2007b, ArXiv Astrophysics e-prints

-. 2010, ApJ, 708, 645

Rykoff, E. S., et al. 2008, ApJ, 675, 1106

Sandage, A., Binggeli, B., \& Tammann, G. A. 1985, AJ, 90, 1759

Scott, D. 1992, John Wiley

Shectman, S. A. 1985, ApJS, 57, 77

Sheldon, E. S., et al. 2007, ArXiv e-prints, 709

Silverman, B. W. 1986, Chapman \& Hall

Smail, I., Edge, A. C., Ellis, R. S., \& Blandford, R. D. 1998, MNRAS, 293, 124

Spergel, D. N., et al. 2003, Astrophys. J. Suppl., 148, 175

—. 2007, Astrophys. J. Suppl., 170, 377

Stott, J. P., Pimbblet, K. A., Edge, A. C., Smith, G. P., \& Wardlow, J. L. 2009, MNRAS, 394, 2098

Tegmark, M., et al. 2004, Phys. Rev. D, 69, 103501 
The Dark Energy Survey Collaboration. 2005, ArXiv Astrophysics e-prints

Visvanathan, N., \& Sandage, A. 1977, ApJ, 216, 214

Yang, X., Mo, H. J., van den Bosch, F. C., Pasquali, A., Li, C., \& Barden, M. 2007, ApJ, 671,153

York, D. G., et al. 2000, AJ, 120, 1579

\section{Acknowledgments}

JH and TM gratefully acknowledge support from NSF grant AST 0807304 and DoE Grant DE-FG02-95ER40899. JH thanks Brian Nord, Jeffery Kubo and Marcelle Soares-Santos for helpful conversation. AEE acknowledges support from NSF AST-0708150 and NASA NNX10AF61G. This work was supported in part by a Department of Energy contract DE-AC02-76SF00515. This project was made possible by workshops support from the Michigan Center for Theoretical Physics.

Funding for the SDSS and SDSS-II has been provided by the Alfred P. Sloan Foundation, the Participating Institutions, the National Science Foundation, the U.S. Department of Energy, the National Aeronautics and Space Administration, the Japanese Monbukagakusho, the Max Planck Society, and the Higher Education Funding Council for England. The SDSS Web Site is http://www.sdss.org/.

The SDSS is managed by the Astrophysical Research Consortium for the Participating Institutions. The Participating Institutions are the American Museum of Natural History, Astrophysical Institute Potsdam, University of Basel, University of Cambridge, Case

This manuscript was prepared with the AAS IATEX macros v5.2. 
Western Reserve University, University of Chicago, Drexel University, Fermilab, the Institute for Advanced Study, the Japan Participation Group, Johns Hopkins University, the Joint Institute for Nuclear Astrophysics, the Kavli Institute for Particle Astrophysics and Cosmology, the Korean Scientist Group, the Chinese Academy of Sciences (LAMOST), Los Alamos National Laboratory, the Max-Planck-Institute for Astronomy (MPIA), the Max-Planck-Institute for Astrophysics (MPA), New Mexico State University, Ohio State University, University of Pittsburgh, University of Portsmouth, Princeton University, the United States Naval Observatory, and the University of Washington. 